\title{
Heterogeneous formation of polar stratospheric clouds - Part 1: Nucleation of nitric acid trihydrate (NAT)
}

\author{
C. R. Hoyle ${ }^{1,2}$, I. Engel ${ }^{2,}$, B. P. Luo ${ }^{2}$, M. C. Pitts ${ }^{3}$, L. R. Poole ${ }^{4}$, J.-U. Grooß ${ }^{5}$, and T. Peter ${ }^{2}$ \\ ${ }^{1}$ Laboratory of Atmospheric Chemistry, Paul Scherrer Institut, Villigen, Switzerland \\ ${ }^{2}$ Institute for Atmospheric and Climate Science, ETH Zurich, Zurich, Switzerland \\ ${ }^{3}$ NASA Langley Research Center, Hampton, Virginia 23681, USA \\ ${ }^{4}$ Science Systems and Applications, Incorporated, Hampton, Virginia 23666, USA \\ ${ }^{5}$ Institut für Energie- und Klimaforschung - Stratosphäre (IEK-7), Forschungszentrum Jülich, Jülich, Germany \\ *now at: Institut für Energie- und Klimaforschung - Stratosphäre (IEK-7), Forschungszentrum Jülich, Jülich, Germany
}

Correspondence to: C. R. Hoyle (christopher.hoyle@env.ethz.ch)

Received: 14 March 2013 - Published in Atmos. Chem. Phys. Discuss.: 22 March 2013

Revised: 16 August 2013 - Accepted: 21 August 2013 - Published: 27 September 2013

\begin{abstract}
Satellite-based observations during the Arctic winter of 2009/2010 provide firm evidence that, in contrast to the current understanding, the nucleation of nitric acid trihydrate (NAT) in the polar stratosphere does not only occur on preexisting ice particles. In order to explain the NAT clouds observed over the Arctic in mid-December 2009, a heterogeneous nucleation mechanism is required, occurring via immersion freezing on the surface of solid particles, likely of meteoritic origin. For the first time, a detailed microphysical modelling of this NAT formation pathway has been carried out. Heterogeneous NAT formation was calculated along more than sixty thousand trajectories, ending at Cloud Aerosol Lidar with Orthogonal Polarization (CALIOP) observation points. Comparing the optical properties of the modelled NAT with these observations enabled a thorough validation of a newly developed NAT nucleation parameterisation, which has been built into the Zurich Optical and Microphysical box Model (ZOMM). The parameterisation is based on active site theory, is simple to implement in models and provides substantial advantages over previous approaches which involved a constant rate of NAT nucleation in a given volume of air. It is shown that the new method is capable of reproducing observed polar stratospheric clouds (PSCs) very well, despite the varied conditions experienced by air parcels travelling along the different trajectories. In a companion paper, ZOMM is applied to a later period of the winter, when ice PSCs are also present, and it is shown that
\end{abstract}

the observed PSCs are also represented extremely well under these conditions.

\section{Introduction}

The discovery of the Antarctic ozone hole (Farman et al., 1985) was rapidly followed by the recognition that polar stratospheric clouds (PSCs), by providing the necessary surface for heterogeneous halogen activation reactions, played a central role in the observed ozone depletion (e.g. Solomon et al., 1986). Solid PSC particles were detected at temperatures well above the frost point, and it was proposed that these particles contained $\mathrm{HNO}_{3}$ (Toon et al., 1986; Crutzen and Arnold, 1986). Later, experimental work suggested that the composition could be that of nitric acid trihydrate (NAT) (Hanson and Mauersberger, 1988), and, more recently, observational work, such as the balloon-borne mass spectrometry measurements reported on by Voigt et al. (2000), have shown that solid PSC particles observed above the frost point do indeed have $\mathrm{H}_{2} \mathrm{O}$ and $\mathrm{HNO}_{3}$ stoichiometries consistent with a NAT composition.

The formation of NAT particles sequesters reactive nitrogen compounds from the gas phase, and because these particles can grow to the large sizes necessary for rapid sedimentation (e.g. Salawitch et al., 1988), they cause denitrification of the polar stratosphere. While it is mostly the liquid particles in the polar stratosphere which are responsible for the heterogeneous reactions leading to the activation of 
the halogens (e.g. Molina et al., 1993; Carslaw et al., 1994; Ravishankara and Hanson, 1996), the observed dimensions of polar ozone loss are only reached because of this denitrification, which prevents deactivation of the halogens.

Despite the importance of NAT particles in determining polar ozone loss, an exact formation mechanism of these particles has remained elusive (e.g. Peter and Grooß, 2012). Initially it was proposed that NAT could crystallise out homogeneously in liquid stratospheric aerosol droplets (Molina et al., 1993; Beyer et al., 1994; Iraci et al., 1994). Later, however, laboratory experiments, using $1 \mathrm{~cm}^{3}$ solution volumes, showed that the homogeneous nucleation rates of NAT in liquid ternary $\left(\mathrm{H}_{2} \mathrm{SO}_{4} / \mathrm{HNO}_{3} / \mathrm{H}_{2} \mathrm{O}\right)$ solution droplets were far too low to lead to the observed formation of such PSCs in the polar stratosphere (Koop et al., 1995). The upper bound that Koop et al. (1995) determined for homogeneous NAT nucleation corresponds to an air-volume-based rate of $J_{\text {NAT }}^{\text {hom, vol,upper }}=1.8 \times 10^{-10} \mathrm{~cm}^{-3}$ air h$^{-1}$, assuming typical stratospheric aerosol particle sizes and number densities. This results in NAT particle number densities lower than $10^{-7} \mathrm{~cm}^{-3}$ when an air mass is cooled below $T_{\mathrm{NAT}}$, the temperature below which NAT is thermodynamically stable, for one week, a period conceivable for the Arctic. Number densities of NAT would still remain below $10^{-6} \mathrm{~cm}^{-3}$, even if air was below $T_{\mathrm{NAT}}$ for 4 months, as might occur in the Antarctic. Such low number densities neither result in effective denitrification, nor are they measurable. Subsequent experiments by the same authors (Knopf et al., 2002), using microlitre size solution volumes, determined an upper bound of $J_{\mathrm{NAT}}^{\text {hom, vol, upper }}=3 \times 10^{-10} \mathrm{~cm}^{-3} \mathrm{air} \mathrm{h}^{-1}$, confirming the results of Koop et al. (1995).

Later, Biermann et al. (1996) showed, based on bulk samples of ternary solution containing solid meteoritic nuclei, that these nuclei could at best have 1/e freezing times of around 20 months. While these authors aimed at excluding meteoritic material as responsible for dense $\left(n>0.1 \mathrm{~cm}^{-3}\right)$ NAT clouds, their derived upper bound corresponds to an airvolume-based rate of $J_{\mathrm{NAT}}^{\text {het, vol, upper }}=7 \times 10^{-4} \mathrm{~cm}^{-3}$ air h$^{-1}$ (assuming a stratospheric smoke surface area density of $5 \mu \mathrm{m}^{2} \mathrm{~cm}^{-3}$ ), which is certainly high enough to produce observable NAT clouds.

In contrast, the nucleation of NAT on ice is very certain and has been demonstrated in the laboratory and in the field. Through a combination of observations and microphysical modelling, Carslaw et al. (1998) and Wirth et al. (1999) showed that NAT can form on ice particles in the atmosphere, suggesting that nucleation of NAT on ice is very efficient and most likely occurs via deposition nucleation on surfaces of ice which are not covered by a liquid coating. Via the so-called "mother cloud/NAT-rock mechanism", Fueglistaler et al. (2002) developed a potential mechanism by which NAT particles initiated by patchy mountain wave ice clouds are distributed vortex-wide.
However, a study by Pagan et al. (2004) showed that during early December 1999, while the Arctic stratosphere was too warm for synoptic ice formation, there was also no ice PSC formation in mountain waves, ruling out nucleation on ice as a formation pathway for the NAT clouds observed during this time period. Drdla et al. (2003) presented modelling work which also suggested that a NAT formation mechanism above the frost point is necessary to explain observed denitrification in the 1999-2000 Arctic winter. Several other studies also exist, where constant NAT nucleation rates have been applied to model NAT formation irrespective of the presence or absence of ice. Using balloon-based measurements carried out in the Arctic in December 2002, Larsen et al. (2004) found that observed PSC properties can be reproduced by applying the surface nucleation rate of Tabazadeh et al. (2002), reduced by a factor of 10-20, to calculate NAD (nitric acid dihydrate) formation from liquid aerosol, followed by instantaneous transformation of NAD to NAT. The rate they determined corresponds to an air-volume-based rate of $J_{\mathrm{NAT}}^{\mathrm{vol}}$ $=2.5 \times 10^{-5} \mathrm{~cm}^{-3} \mathrm{airh}^{-1}$. The air masses which they observed had not been exposed to synoptic temperatures below the frost point, and there was no mountain wave activity; therefore NAT formation on ice could be ruled out, however heterogeneous nucleation on other types of solid particles could not be excluded.

More recently, Voigt et al. (2005) reported aircraft observations of low number densities of NAT particles in air masses which had been at $T>T_{\mathrm{NAT}}+10 \mathrm{~K}$ some 24 $36 \mathrm{~h}$ before the measurement and then cooled to $T_{\mathrm{NAT}}>$ $T>T_{\text {ice }}+3 \mathrm{~K}$ for a period of less than $20 \mathrm{~h}$ before the measurement. In order to explain these observations, they suggested that NAT may also nucleate heterogeneously on the surface of meteoritic dust particles. Because the time below $T_{\text {NAT }}$ could be well constrained $(20 \mathrm{~h})$, they were able to estimate a volume-based NAT nucleation rate of $J_{\text {NAT }}^{\text {het, vol }}=8 \times 10^{-6} \mathrm{~cm}^{-3}$ air h$^{-1}$, reproducing their observation of NAT number densities of $1.6 \times 10^{-4} \mathrm{~cm}^{-3}$. While this rate reproduced the observed number densities, because of the limited measurement data, it was not possible to determine how the NAT nucleation rate increases as temperature decreases below $T_{\mathrm{NAT}}$. It was argued by Voigt et al. (2005) that the heterogeneous NAT nucleation rates on meteoritic material measured by Biermann et al. (1996), while too low to be responsible for dense NAT clouds, might actually be sufficient to produce low NAT number densities within a few hours. Since the nucleation rates published by Biermann et al. (1996) were upper limits and the real rates might be considerably smaller, the suitability of meteoritic dust remains an open issue, which calls for further investigation.

Also for the Arctic winter of 2002/2003, Grooß et al. (2005) found that using a constant volume-based rate of $J_{\text {NAT }}^{\text {vol }}=8 \times 10^{-6} \mathrm{~cm}^{-3}$ air h$^{-1}$ in CLaMS (Chemical Lagrangian Model of the Stratosphere), led to the best agreement between modelled and observed PSC properties and 
Table 1. NAT nucleation rates from previous studies, as described in the text. All rates except for $f\left(S_{\mathrm{NAT}}\right)$ are air-volume-based, and lead to NAT nucleation as soon as the temperature drops below $T_{\text {NAT }}$.

\begin{tabular}{ll}
\hline Nucleation rate & Reference \\
\hline$J_{\text {NAT }}^{\text {hom, vol,upper }}=1.8 \times 10^{-10} \mathrm{~cm}^{-3}$ air h$^{-1}$ & Koop et al. (1995) \\
$J_{\text {NAT }}^{\text {hom, vol,upper }}=3.0 \times 10^{-10} \mathrm{~cm}^{-3}$ air h$^{-1}$ & Knopf et al. (2002) \\
$J_{\text {NAT }}^{\text {het, vol,upper }}=7.0 \times 10^{-4} \mathrm{~cm}^{-3}$ air h & Biermann et al. (1996) \\
$J_{\text {NAT }}^{\text {vol }}=2.9 \times 10^{-6} \mathrm{~cm}^{-3}$ air h & Carslaw et al. (2002) \\
$J_{\text {NAT }}^{\text {vol }}=2.5 \times 10^{-5} \mathrm{~cm}^{-3}$ air h & Larsen et al. (2004) \\
$J_{\text {NAT }}^{\text {het, vol }}=8.0 \times 10^{-6} \mathrm{~cm}^{-3}$ air h$^{-1}$ & Voigt et al. (2005) \\
$J_{\text {NAT }}^{\text {vol }}=8.0 \times 10^{-6} \mathrm{~cm}^{-3}$ air h & Grooß et al. (2005) \\
$J_{\text {NAT }}^{\text {het, vol }}=9.0 \times 10^{-6} \mathrm{~cm}^{-3}$ air h $^{-1}$ & This work \\
$J_{\text {NAT }}^{\text {het }}=f\left(S_{\mathrm{NAT}}\right)$ & This work \\
\hline
\end{tabular}

1 The volume-based nucleation rate used in this work, for comparison with the newly developed parameterisation.

2 The newly developed parameterisation presented here is not air-volume-based, and allows for a saturation dependence of the NAT nucleation rate.

denitrification throughout the winter. A domain-filling, Lagrangian particle trajectory model was used by Carslaw et al. (2002) to simulate the characteristics of NAT particles in the Arctic stratosphere in January-March 2000. They also used a simple volume-based NAT nucleation rate, and found that the observed particle sizes were well reproduced when a rate of $J_{\text {NAT }}^{\mathrm{vol}}=2.9 \times 10^{-6} \mathrm{~cm}^{-3}$ air h${ }^{-1}$ was used. While the apparent patchiness of wave ice clouds was first taken as an argument against their effectiveness in NAT nucleation, later more detailed vortex-wide simulations by the same authors showed that this mechanism could potentially indeed explain up to $80 \%$ of the observed denitrification (Mann et al., 2005). Davies et al. (2005) simulated Arctic denitrification using a combination of a Lagrangian particle model and the chemistry transport model SLIMCAT. They compared the modelled denitrification with $\mathrm{HNO}_{3}$ measurements from satelliteand aircraft-based instruments, and found reasonable agreement for several winters, when using the same NAT nucleation rate as Carslaw et al. (2002). However, for the winter of 1994/1995, they found that in order to reproduce the observed denitrification, this nucleation rate needs to be increased by a factor of 4 , highlighting the need for physically based nucleation rates. The various air-volume-based NAT nucleation rates from previous studies are summarised in Table 1.

During December 2009, space-borne lidar measurements conducted from CALIPSO (Cloud-Aerosol Lidar and Infrared Pathfinder Satellite Observations), showed a situation where thin and patchy liquid-NAT mixed clouds of unprecedented dimensions (typically $1000 \times 2000 \mathrm{~km}^{2}$ ) were present in air masses in the Arctic, despite these air masses not hav- ing experienced temperatures below the frost point (Pitts et al., 2011). In the present study, this observational data set is utilised to develop a physically based parameterisation for NAT nucleation on solid, non-ice particles. The advantage of using such satellite-based lidar observations is that they cover a large area of the Arctic vortex, and when back trajectories are calculated starting from the observations, a range of air parcel histories are found, allowing the development of a robust parameterisation, which represents NAT formation in air reaching different supersaturations with respect to NAT, for different lengths of time.

In the next section, the observational data set is described, as well as the models which were applied, and the new NAT nucleation parameterisation. In Sect. 3, the results of modelling NAT formation with the new parameterisation are presented and compared with a constant nucleation rate approach, and in Sect. 4 we provide a summary and conclusions of the study. In a companion paper (Part 2; Engel et al., 2013) the role of heterogeneous nucleation in ice formation is discussed.

\section{Observational data and model description}

\subsection{The CALIPSO observations}

The CALIPSO satellite was launched in April 2006, and includes the Cloud Aerosol Lidar with Orthogonal Polarization (CALIOP) as its principal instrument. CALIOP measures backscatter at two wavelengths, $1064 \mathrm{~nm}$ and $532 \mathrm{~nm}$, using the $532 \mathrm{~nm}$ signal to measure the depolarisation of the backscattered radiation relative to the plane-polarised light of the outgoing laser beam. With its high-latitude coverage up to $82^{\circ}$, CALIOP has provided extensive observations of PSCs in both hemispheres since its launch.

In order to derive and test the new NAT nucleation parameterisation, the CALIOP observations made over the Arctic in December 2009 are used. During the second half of December 2009, stratospheric temperatures were slightly below $T_{\mathrm{NAT}}$ and patchy areas of PSCs were detected on a daily basis. The vortex average temperatures were several degrees above $T_{\text {frost }}$ (Dörnbrack et al., 2012), and, additionally, there were no observations of ice clouds during this period. In Fig. 1, the minimum temperatures with respect to the frost point, along all trajectories used in the analysis here (as described in Sects. 2.3 and 3), are shown. Even the lowest temperatures along these trajectories are $2.5 \mathrm{~K}$ above the frost point, while the peak of the distribution is at approximately $5 \mathrm{~K}$ above the frost point. Considering that temperatures approximately $3 \mathrm{~K}$ below the frost point are required to homogeneously nucleate ice, even accounting for small-scale temperature fluctuations, the probability of undetected ice clouds having occurred is extremely low. This set of observations and model calculations therefore supports the existence of a NAT nucleation mechanism which does not require an ice 


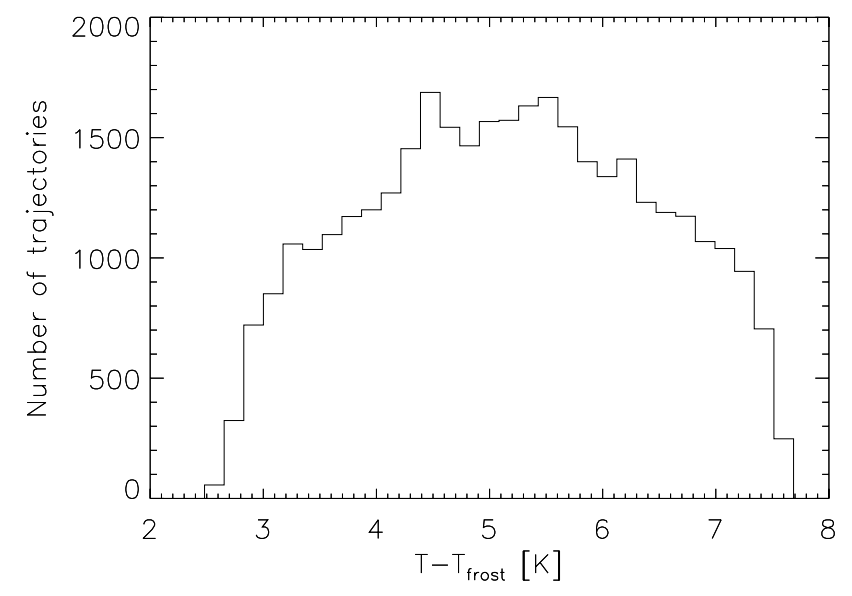

Fig. 1. A distribution showing the minimum temperature reached along each trajectory, with respect to the frost point. Trajectories were calculated back in time from points along the CALIPSO orbit path, as described in Sect. 2.3. All trajectories used to model the PSC properties along all orbits in Sect. 3 are included in the figure.

surface. On 31 December, mountain wave ice clouds were first observed, followed by a dramatic increase in observations of NAT-containing clouds, marking the end of the period when the NAT PSCs can be considered to have formed without the presence of ice particles.

In the analysis here, we make use of the PSC classification system of Pitts et al. $(2009,2011)$, which assigns the detected clouds to one of six categories (STS, Mix1, Mix2, Mix2-enh, ice, wave ice), depending on the backscatter and depolarisation signals. Though not identical, this classification system is similar to the previously used PSC types, namely $1 \mathrm{~b}$ (STS), 1a (Mix1, Mix2), 1a-enh (Mix2-enh), and 2 (ice and wave ice). For the NAT classes, Mix1 includes NAT number densities of less than approximately $10^{-3} \mathrm{~cm}^{-3}$, Mix 2 includes NAT number densities between about $10^{-3} \mathrm{~cm}^{-3}$ and $0.1 \mathrm{~cm}^{-3}$, while Mix2-enh includes NAT number densities of above $0.1 \mathrm{~cm}^{-3}$.

The PSC detection limit of CALIOP is altitude dependent (Pitts et al., 2009), and we treat the modelled data in a similar way. If the modelled or observed values at a particular point are below the detection limit, then a larger horizontal averaging distance is applied. Thus, for the observations, if no PSC is detected over $5 \mathrm{~km}$, the horizontal resolution is decreased (i.e. the averaging distance is increased) to $15 \mathrm{~km}$, then $45 \mathrm{~km}$ and $135 \mathrm{~km}$. The highest resolution for the modelled data is $25 \mathrm{~km}$ (the resolution of the grid from which the trajectories used by the model were started), and if no PSCs are detected at a point then the averaging distance is increased to $125 \mathrm{~km}$.

\subsection{The microphysical box model}

The Zurich Optical and Microphysical box Model (ZOMM) used in this study has been applied to simulate PSCs (e.g. Meilinger et al., 1995; Luo et al., 2003) as well as cirrus clouds (e.g. Hoyle et al., 2005; Brabec et al., 2012). In the latter two studies, a slightly different version of the model was used; however the only significant difference is that the stratospheric version of the model simulates the evolution of a population of ternary solution aerosol droplets $\left(\mathrm{H}_{2} \mathrm{O}\right.$, $\mathrm{H}_{2} \mathrm{SO}_{4}, \mathrm{HNO}_{3}$ ) and their interaction with the gas phase, whereas the tropospheric version accounts for a binary $\left(\mathrm{H}_{2} \mathrm{O}\right.$, $\mathrm{H}_{2} \mathrm{SO}_{4}$ ) solution.

The most prominent feature of the model is that it treats particle sizes in a Lagrangian scheme; i.e. the model follows the radii of growing/shrinking particles and creates a new size class $i$ each time there is new ice or NAT particle nucleation. The model then transports the liquid, NAT and ice particles size class $i$ downstream with time-dependent radius $r_{i}(t)$ and constant number density, $n_{i}$. The Lagrangian size treatment avoids numerical diffusion, which may lead to artificial redistributions of particle number densities on a fixed Eulerian size grid with fixed bins.

The model is initialised with a log-normally distributed population of solution droplets, which represent the background aerosol. This initial distribution is calculated given a user-defined mode radius, number density and standard deviation, and in this study consisted of 26 radii. The initial composition of the droplets is calculated assuming thermodynamic equilibrium with the gas phase and accounting for the Kelvin effect of the various species. The total mixing ratios of $\mathrm{H}_{2} \mathrm{O}, \mathrm{H}_{2} \mathrm{SO}_{4}$ and, in the PSC version, $\mathrm{HNO}_{3}$, are set in the input file and are assumed to remain constant for the whole simulation along a trajectory (i.e. neglecting mixing and particle sedimentation).

The model is run along a trajectory consisting of a time series of temperature and pressure data points, and after the initialisation, the aerosol droplet sizes are allowed to evolve freely in radius space, and are no longer restricted to a lognormal form (see Meilinger et al., 1995). As the temperature decreases, growth of the aerosol droplets is calculated, fully kinetically for all species, whereby the Kelvin effect is accounted for in calculating the vapour pressures of the individual species over the droplet surfaces. Latent heat effects of condensation and evaporation (or freezing) are not accounted for in the PSC version of the model; however given the small mixing ratios of matter which condense or evaporate (a few ppmv for $\mathrm{H}_{2} \mathrm{O}$ and a few ppbv for $\mathrm{HNO}_{3}$ ) in the polar stratospheres, this does not impart any significant uncertainties to the calculations. Accommodation coefficients for the uptake/evaporation of species from/to the gas phase were set to 1.0, for liquid and for solid particles.

A schematic representation of the modelled composition and phase changes of PSCs as a function of temperature is shown in Fig. 2. Pathways previously represented in ZOMM are marked with dashed arrows; those which have been added for this study are shown by solid arrows. At temperatures above about $200 \mathrm{~K}$, supercooled aerosol droplets exist as a quasi-binary $\mathrm{H}_{2} \mathrm{O} / \mathrm{H}_{2} \mathrm{SO}_{4}$ solution. Upon further cooling, $\mathrm{HNO}_{3}$ is taken up to form a supercooled ternary solution 


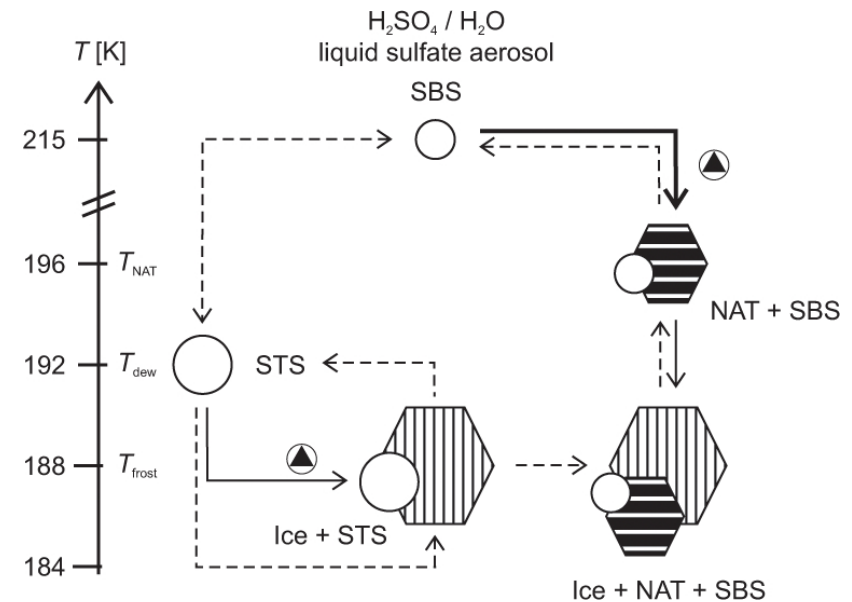

Fig. 2. A schematic description of the different pathways included in ZOMM. The dashed arrows depict processes which were represented in previous model versions; the solid arrows show those added to the model for this study. $T_{\text {dew }}$ denotes the dew point, i.e. the temperature at which water saturation is reached. The temperatures indicated for $T_{\mathrm{dew}}, T_{\text {frost }}$ and $T_{\mathrm{NAT}}$ are approximate, based on typical polar stratospheric conditions. Foreign nuclei are indicated by the black triangles. While here the focus is on the heterogeneous nucleation of NAT (thick black arrow), the heterogeneous nucleation of ice (thin solid black arrows) is discussed in a companion paper (Engel et al., 2013). The heterogeneous nucleation of NAT occurs in STS aerosol droplets which contain a foreign nucleus (triangle surrounded by circle on the upper right of the figure), after the nucleation of NAT and as the air parcel continues to cool, a supercooled binary solution (SBS) coating, consisting of $\mathrm{H}_{2} \mathrm{O}$ and $\mathrm{H}_{2} \mathrm{SO}_{4}$, is present on the NAT particle.

(STS). In the absence of solid nuclei, ice may nucleate homogeneously in the STS droplets when temperatures decrease a few degrees below the frost point, and NAT can then form on the surface of the ice particles. Alternatively, if foreign nuclei are present (as indicated by the black triangles), they may lead to ice nucleation at higher temperatures than in the homogeneous case (Engel et al., 2013), or, as shown here, to the formation of NAT in the absence of ice (thick black line in Fig. 2).

\subsubsection{Homogeneous ice nucleation}

The homogeneous nucleation rate coefficient for the formation of water ice, $J_{\text {ice }}^{\text {hom }}$, is calculated based on the temperature and the water activity in the droplet according to

$J_{\text {ice }}^{\text {hom }}=10^{D} \mathrm{~cm}^{-3} \mathrm{~s}^{-1}$,

where

$D=-906.688+8502.28 x-26924.4 x^{2}+29179.6 x^{3}$

and $x=a_{\mathrm{w}, \text { liq }}-a_{\mathrm{w} \text {,ice }}$ is the water activity in a droplet $\left(a_{\mathrm{w}, \mathrm{liq}}\right)$ minus the water activity in a solution which would be in equilibrium with ice $\left(a_{\mathrm{w}, \text { ice }}\right)$ at the particular temperature being considered. $a_{\mathrm{w} \text {,ice }}$ is defined as the ratio of the $\mathrm{H}_{2} \mathrm{O}$ vapour pressures over ice and pure water, and its value is given by Zobrist et al. (2011), based on Koop et al. (2000), as

$a_{\mathrm{w}, \text { ice }}=$

$\exp \left(-\frac{-210368-131.438 T+3.32373 \times 10^{6} / T+41729.1 \times \ln (T)}{8.31441 \times T}\right)$,

where $T$ is the absolute temperature in Kelvin. The expected number density of ice particles $\left(n_{\text {ice }}\right)$ nucleated from modelled liquid droplets with number density $n_{\text {liq }}$ and volume $v_{\text {liq }}$ can be calculated by

$n_{\text {ice }}=n_{\text {liq }}\left(1-\exp \left(-\int_{t_{0}}^{t} J_{\text {ice }}^{\text {hom }} v_{\text {liq }} \mathrm{d} t\right)\right)$.

Instead of forming a new Lagrangian ice particle size class for every droplet size class, in every time step, which would be computationally impractical, the number of nucleated ice particles in each droplet size class is accumulated until this number exceeds either $1 \times 10^{-5} \mathrm{~cm}^{-3}$, or half the number density of droplets in that size class, or half of the total number density of ice particles in the model, whichever of the three criteria is satisfied first.

After ice is nucleated in a liquid size class, the number of frozen droplets calculated using Eq. (4) is transferred from the liquid size class to the new ice size class. All ice particles in a particular size class thus have the same radius, and this radius increases as the ice particles take up water in competition with the remaining liquid droplets. Upon warming, the evaporation/sublimation of species from the solid particles is calculated, until eventually a concentrated solution droplet is released and the number of particles is transferred back from the ice size class to the liquid size class from where it originated.

\subsubsection{NAT formation}

Until now, NAT formation in ZOMM was only permitted via deposition nucleation on clean (uncoated, dry) surfaces of ice particles. The method used was thoroughly described by Luo et al. (2003); a summary of this description is provided here.

Ice particles in the atmosphere are generally coated in a layer of liquid solution; however there may be dry patches on the particle, where the ice interacts directly with the gas phase. In rapid cooling events, the supersaturations with respect to NAT in the gas phase can grow to much larger values than in the liquid due to non-equilibrium effects, favouring deposition nucleation on these exposed clean ice surfaces (Luo et al., 2003). The area of the dry patches was determined by assuming a minimum thickness of the liquid layer of $28.5 \times 10^{-8} \mathrm{~cm}$ (approximately 10 monolayers; Luo et al., 2003), and calculating the resulting surface coverage of the actual volume of liquid available to form the layer on the solid particles. The NAT nucleation rate coefficient on the 
dry area of the ice particles was calculated based on the classical nucleation theory for the heterogeneous nucleation of water ice, using Eq. (5):

$$
\begin{aligned}
J_{\mathrm{NAT}}^{\text {het, ice }}(T) & =\frac{k T}{h} \exp \left[-\frac{\Delta F_{\text {diff }}(T)}{k T}\right] \\
& \times n \exp \left[-\frac{\Delta G(T) f_{\text {het }}(\alpha)}{k T}\right],
\end{aligned}
$$

where $T$ is the temperature in Kelvin, $k$ and $h$ the Boltzmann and Planck constants respectively, $n$ is the number density of $\mathrm{HNO}_{3}$ molecules on the ice surface (ca. $2 \times 10^{14} \mathrm{~cm}^{-2}$, at high NAT supersaturations; see Abbatt, 1997), $\Delta F_{\text {diff }}$ is the temperature-dependent diffusion activation energy which has to be surmounted for 2-D diffusion of $\mathrm{HNO}_{3}$ to take place on the surface of a preexisting foreign nucleus (here the ice), and $G$ is the Gibbs free energy for the formation of a critical NAT embryo in the absence of the foreign nucleus, i.e.

$\Delta G(T)=\frac{16 \pi}{3} \frac{m^{2} \sigma^{3}}{\left[k T \rho \ln \left(S_{\mathrm{NAT}}\right)\right]^{2}}$.

In Eq. (6), $m$ is the molecular mass of NAT, $\sigma$ is the surface energy of NAT, and $S_{\mathrm{NAT}}$ is the NAT saturation ratio. The presence of the foreign nucleus reduces $\Delta G$ by the compatibility factor $f_{\text {het }}$, defined as

$f_{\text {het }}(\alpha)=\frac{1}{4}(2+\cos \alpha)(1-\cos \alpha)^{2}$,

where $\alpha$ is the contact angle, a parameterisation of the heterogeneous nucleation activity of the foreign nucleus (Seinfeld and Pandis, 2006). The possible values for $\alpha$ range between $0^{\circ}$ and $180^{\circ}$, leading to values of $f_{\text {het }}$ between 0 and 1 ; i.e. with a contact angle of $180^{\circ}$ the foreign nucleus does not cause any enhancement in the nucleation rate.

Now, as $\Delta F_{\text {diff }}$ is unknown, as are the compatibility factor $f_{\text {het }}$ and the surface energy of NAT, Luo et al. (2003) defined two new constants (denoted here as $\mathrm{b}$ and $\gamma$ ), which they used to tune their nucleation rate coefficient equation, so that the model reproduced the observed NAT distribution:

$$
\begin{aligned}
& J_{\mathrm{NAT}}^{\text {het,ice }}=6.24 \times 10^{24} \mathrm{~cm}^{-2} \mathrm{~s}^{-1} \mathrm{~K}^{-1} \\
& T \exp \left[\frac{-b}{T}\right] \exp \left[-\frac{\gamma(273.15)^{3}}{T^{3}\left(\ln \left(S_{\mathrm{NAT}}\right)\right)^{2}}\right],
\end{aligned}
$$

where the value $6.24 \times 10^{24}$ corresponds to $k n / h$. The values that Luo et al. (2003) suggested for these two constants were

$b=\frac{\Delta F_{\text {diff }}}{k}=2000 \mathrm{~K}$

and

$\gamma=\frac{\Delta G(T) T^{2} \ln \left(S_{\mathrm{NAT}}(T)\right)^{2} f_{\text {het }}(\alpha)}{273.15^{3} k}=328 \mathrm{~K}^{3}$.

Note that in this parameterisation, it is implicitly assumed that the nucleation sites on the foreign nuclei are all of the same quality, as the compatibility factor is included in $\gamma$ and is therefore invariant.

\subsubsection{Extension to heterogeneous NAT nucleation and heterogeneous ice nucleation on foreign nuclei}

The microphysical box model has recently been extended so that in addition to homogeneous nucleation of water ice, and heterogeneous nucleation of NAT on the surface of ice particles, it now includes the heterogeneous nucleation of ice on foreign nuclei as well as NAT formation on foreign nuclei (both in the immersion mode). These two new nucleation pathways are based on the active site parameterisation of Marcolli et al. (2007). As foreign nuclei, a monodisperse population of solid particles with radii of $20 \mathrm{~nm}$ and with a number density of $7.5 \mathrm{~cm}^{-3}$ is assumed. Measurements reported by Curtius et al. (2005) show that during January to March 2003 approximately $67 \%$ of aerosol particles in the Arctic polar vortex contained non-volatile residuals, while outside the vortex the value was much lower, at approximately $24 \%$. Similar values (between $30 \%$ and $70 \%$ depending on altitude above the tropopause) are reported for meteoritic particles in aerosol by Murphy et al. (2007), for mid- to low latitudes. During the RECONCILE (Reconciliation of essential process parameters for an enhanced predictability of Arctic stratospheric ozone loss and its climate interactions) campaign, values of up to $80 \%$ were found (von Hobe et al., 2013). The value used here, of $7.5 \mathrm{~cm}^{-3}$ aerosol particles containing foreign nuclei, corresponding to $50 \%$ of the total aerosol, was chosen as a conservative estimate. As discussed below, only a small fraction of these nuclei actually participate in the NAT nucleation; therefore there would only be a significant effect on the results presented here if a far lower percentage were to be chosen.

According to the active site theory, freezing cannot be initiated equally well over the entire surface of the foreign nucleus; rather it occurs preferentially at certain active sites. As a proxy for the quality of these sites, the contact angle may be used, which essentially parameterises the reduction in the nucleation energy barrier associated with a particular active site. In applying Eq. (5) to NAT nucleation on nuclei other than clean ice surfaces, the variations in contact angle for active sites of different quality must be accounted for. We therefore define $\gamma^{\prime}$, which differs from $\gamma$ of Luo et al. (2003) only in that it no longer includes the compatibility factor. The new parameterisation for the nucleation rate coefficient of NAT, on foreign nuclei immersed in a ternary $\mathrm{H}_{2} \mathrm{O} / \mathrm{HNO}_{3} / \mathrm{H}_{2} \mathrm{SO}_{4}$ solution, is therefore

$$
\begin{aligned}
J_{\text {NAT }}^{\text {het,foreign }}(T) & =6.24 \times 10^{24} \mathrm{~cm}^{-2} \mathrm{~s}^{-1}(T / K) \exp \left[\frac{-b}{T}\right] \\
& \times \exp \left[-\frac{\gamma^{\prime}(273.15)^{3} f_{\text {het }}(\alpha)}{T^{3}\left(\ln \left(S_{\mathrm{NAT}}\right)\right)^{2}}\right],
\end{aligned}
$$

where $S_{\mathrm{NAT}}$ is the saturation ratio of the STS with respect to NAT.

In order to adjust the nucleation rate parameterisation so that modelled NAT distributions correspond to observations, the value of $\gamma^{\prime}$ and the distribution of contact angles $\alpha$ for 
the available active sites on the foreign nuclei must be determined. Assuming that only the best active site on a particle is responsible for NAT nucleation, the occurrence probability of an active site with a particular contact angle on a particle can be described as

$P_{\text {as }}(\alpha)=P_{\text {pre }} \times \exp \left(\frac{-51.0^{\circ}}{\alpha-\alpha_{0}}\right)$

similarly to Marcolli et al. (2007). The parameter $\alpha_{0}$ represents the lowest contact angle considered, while $P_{\text {pre }}$ is a constant (with units of $\mathrm{deg}^{-1}$ ). In the model, $1^{\circ}$ contact angle bins were used, for contact angles starting from $\alpha_{0}+1^{\circ}$. The number density of foreign nuclei, containing at least one active site with a minimum contact angle $\alpha$ (i.e. the number of foreign nuclei in one particular contact angle bin), is then given by

$n_{\alpha}=\left(n_{\text {foreign }}-\sum_{\alpha^{\prime}=\alpha_{0}+1}^{\alpha-1} n_{\alpha^{\prime}}\right) P_{\text {as }}(\alpha) \Delta \alpha \frac{4 \pi r_{\text {foreign }}^{2}}{A_{1}}$,

where $\Delta \alpha=1^{\circ}, r_{\text {foreign }}$ is the radius of the foreign nuclei ( $20 \mathrm{~nm}$ ) and $A_{1}$ is the area of one active site, which is taken to be $10 \mathrm{~nm}^{2}$, following Marcolli et al. (2007). The nucleation rate of NAT on a particle is then $J_{\mathrm{NAT}}^{\text {het, foreign }}$ multiplied by the area of the active site, $A_{1}$. This corresponds to a volumebased nucleation rate of

$J_{\mathrm{NAT}}^{\text {het, vol,foreign }}(T)=\sum_{\alpha_{i}=\alpha_{0}+1}^{\alpha_{\max }} n_{\alpha_{i}} \times J_{\mathrm{NAT}}^{\text {het,foreign }}(T, \alpha) \times A_{1}$.

The number density of foreign nuclei as a function of the contact angle of their best active site is shown in Fig. 3. This figure highlights the contrast between the very few excellent nuclei, characterised by small contact angle active sites, and the large number of mediocre nuclei with larger contact angles. Similar distributions of nuclei quality have previously been observed for populations of volcanic ash particles (Hoyle et al., 2011) and mineral dust particles (Pinti et al., 2012) in ice nucleation experiments. The curve in Fig. 3 becomes rather flat beyond $80^{\circ}$, indicating there are similar numbers of particles having a best active site with a contact angle of, for example, $100^{\circ}$ or $120^{\circ}$. With a foreign nuclei number density of $7.5 \mathrm{~cm}^{-3}$, and setting $P_{\text {pre }}=$ $1 \times 10^{-6} \mathrm{deg}^{-1}$ (see below), considering contact angles up to $146^{\circ}$ accounts for approximately 0.13 particles $\mathrm{cm}^{-3}$, which covers the range of NAT number densities necessary for simulating the PSCs observed during the winter of 2009/2010. Note that Eq. (12) is not normalised, which effectively means that the majority of the foreign nuclei have best active sites which are of insufficient quality to decrease the NAT nucleation barrier.

The properties of the PSC modelled with Eq. (11) can be adjusted by varying the values of the three fitting parameters $\gamma^{\prime}, \alpha_{0}$ and $P_{\text {pre. }}$. A higher value of $\gamma^{\prime}$ effectively increases the nucleation barrier represented by the last term of Eq. (11),

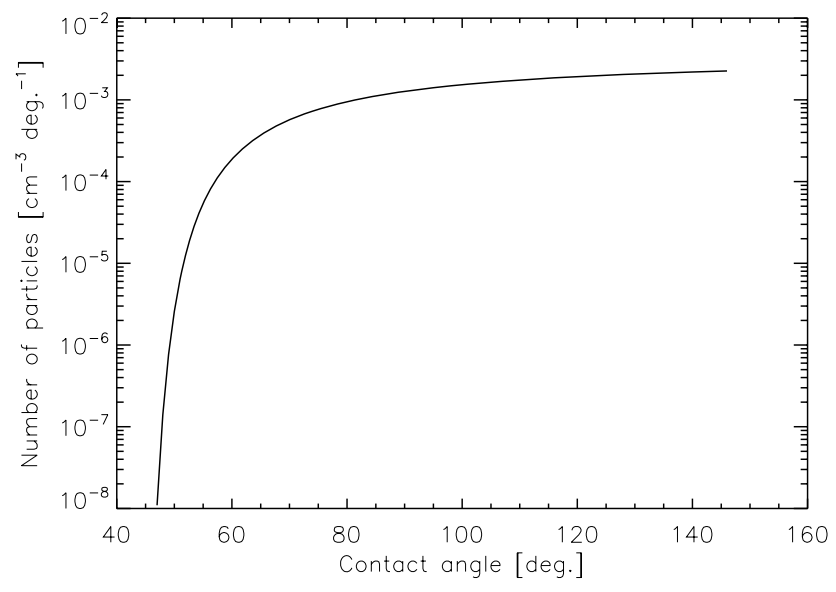

Fig. 3. The number density of foreign nuclei as a function of the contact angle of the best active site on the particle's surface.

and therefore lowers the temperature at which NAT will first form. The parameter $\alpha_{0}$ sets a lower limit on the contact angle associated with the best possible active site. As described above, an active site with a small contact angle has a low value of the compatibility factor $\left(f_{\text {het }}\right)$; therefore $\alpha_{0}$ determines the maximum extent to which the presence of a foreign nucleus can reduce the nucleation barrier. On the other hand, $P_{\text {pre }}$ changes the probability of an active site with a particular contact angle occurring, and therefore alters the number density of NAT that can be formed at a particular temperature. As it is assumed that the best active site on a foreign nucleus is responsible for the nucleation event, an increase in $P_{\text {pre }}$ shifts the distribution of active sites towards lower contact angles, increasing the number of NAT particles which nucleate at higher temperatures.

While for ice the nucleation rate is the sum of the heterogeneous and homogeneous rates, in the case of NAT formation only the heterogeneous nucleation rate on foreign nuclei and on ice is considered. Upon evaporation of the ice or NAT particles, the nuclei are returned to the active site bin from which they originated.

The choice of the fitting variables for NAT nucleation, as well as the other parameters used in this particular study, is discussed in Sect. 2.3, while the parameters used for heterogeneous ice nucleation are given in Engel et al. (2013).

The optical properties (backscatter ratio, $R_{532}$, and aerosol depolarisation ratio, $\delta_{\text {aerosol }}$ ) of the modelled PSC are calculated from the particle number densities and radii, assuming the particles to be prolate spheroids with aspect ratios (diameter-to-length ratios) of 0.9 (in accordance with Carslaw et al., 1998) and a refractive index of 1.48 for NAT (Middlebrook et al., 1994). Calculations were then made using a T matrix light scattering algorithm (Mishchenko, 1991; Carslaw et al., 1998) for spheroids with a known refractive index. 

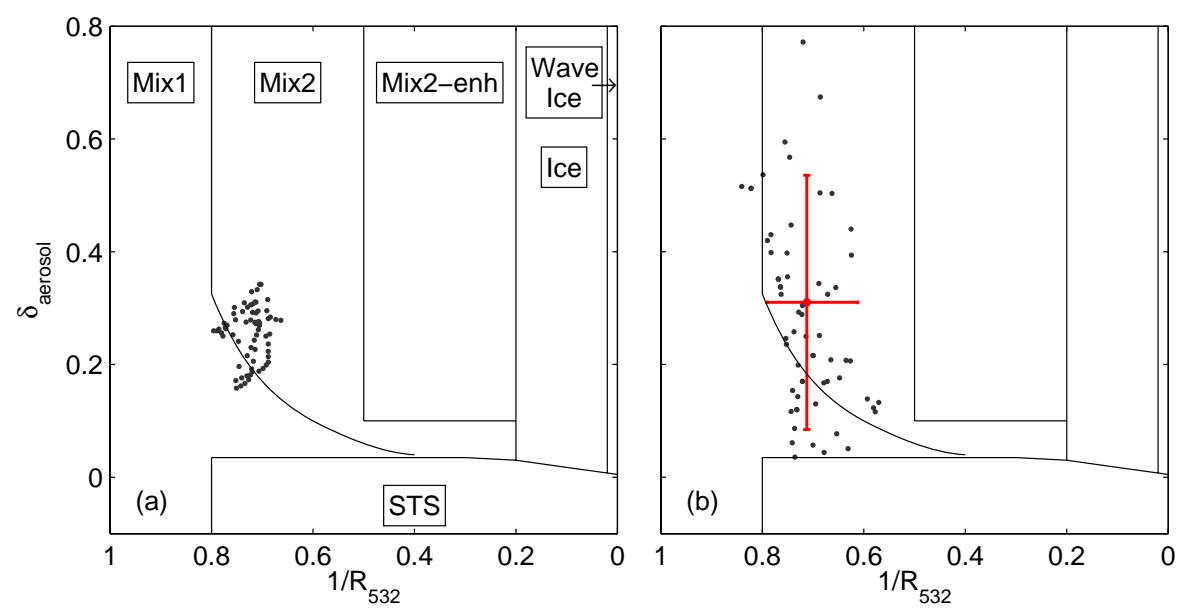

Fig. 4. Modelled aerosol depolarisation ratio $\left(\delta_{\text {aerosol }}\right)$ vs. inverse backscatter ratio $\left(1 / R_{532}\right)$ without (panel a) and with (panel b) consideration of instrument noise, for the mixed NAT/liquid cloud observed on the third CALIPSO overpass of the Arctic vortex on 21 December (denoted here as orbit 21_03). The error bars illustrate one standard deviation of the uncertainty for a single point. The different areas of the plots correspond to the PSC classifications described in Sect. 2.1.

The CALIOP measurements of parallel and perpendicular backscatter are subject to an uncertainty, and to simulate this in the model results, a normally distributed random instrumental noise component is added to each of these quantities in the model output, before calculating $R_{532}$ and $\delta_{\text {aerosol }}$. Subsequently, the modelled PSC is classified according to the same scheme used for the observations. This allows a direct comparison between the modelled and observed PSC properties. The calculation of the uncertainty in the CALIOP measurements is described in detail in Engel et al. (2013), and an example of the model output without and with the instrumental noise is shown in Fig. 4, panels a and b respectively. Additionally, error bars show the limits of one standard deviation of the normally distributed noise component for a single modelled point. The main effect of the random noise is to increase the variability in the depolarisation ratio.

\subsection{Trajectory calculations}

The microphysical model was run along trajectories which ended at CALIPSO observation points. For every CALIPSO orbit during December 2009, where PSCs were observed, the following criteria were applied to choose the observation points used in the analysis:

- Latitude north of $50^{\circ} \mathrm{N}$;

- Altitude of between $18 \mathrm{~km}$ and $26 \mathrm{~km}$;

- The temperature at the observation point was $T_{\mathrm{NAT}}$ or less;

- Every 5th vertical profile available in the CALIPSO data set was chosen, to give a $25 \mathrm{~km}$ horizontal resolution;
- Every vertical level was used, providing approximately a $180 \mathrm{~m}$ vertical resolution.

From all the points fulfilling these criteria, ten-day backward trajectories were calculated, using the trajectory module of CLaMS (McKenna et al., 2002). The trajectories were based on ECMWF ERA-Interim data (Dee et al., 2011) with a resolution of $1^{\circ} \times 1^{\circ}$ and $6 \mathrm{~h}$, using potential temperature as the vertical coordinate. The vertical resolution was approximately $1.25 \mathrm{~km}$ between the altitudes of $19 \mathrm{~km}$ and $30 \mathrm{~km}$ at polar latitudes. The internal and output time step for the trajectory calculation was $15 \mathrm{~min}$. To determine the starting point for the model calculation, the trajectories were followed backward in time from their end points, until the temperature rose above $T_{\mathrm{NAT}}$, so as to avoid the complication of having to initialise the PSCs. If no such point was encountered within the ten days preceding the CALIPSO measurement on which the trajectory ended, the trajectory was not used in the modelling. For each of the valid trajectories, the model was initialised and run forward in time up until the point of the CALIPSO observation. For the days which were investigated in December, there were around 20005000 valid trajectories per orbit.

In previous work on cirrus nucleation (e.g. Kärcher and Lohmann, 2002; Hoyle et al., 2005), as well as in the analysis of ice PSCs (Engel et al., 2013), it has been shown that the observed microphysical properties of a cloud can only be properly reproduced by a model if small-scale temperature fluctuations (for example resulting from gravity waves with horizontal wavelengths of less than $100 \mathrm{~km}$, i.e. below the resolution of CLaMS and the ERA-Interim data) are accounted for. Such fluctuations are expected to be ubiquitous in the atmosphere (e.g. Gary, 2006, 2008) and have been the subject of numerous PSC studies in the past, most of which 
examine the formation of ice and subsequent NAT nucleation on ice particles (e.g. Fueglistaler et al., 2002; Luo et al., 2003; Mann et al., 2005; Höpfner et al., 2006; Eckermann et al., 2009; Alexander et al., 2011).

It was demonstrated by Meilinger et al. (1995) that, as the equilibration times for $\mathrm{HNO}_{3}$ and for $\mathrm{H}_{2} \mathrm{O}$ in droplets differ greatly, during the warming phase of a temperature fluctuation, $\mathrm{H}_{2} \mathrm{O}$ may partition from a liquid droplet back into the gas phase much more quickly than $\mathrm{HNO}_{3}$. This can result in an enhanced NAT saturation ratio, and NAT nucleation at higher temperatures than would otherwise be possible in the absence of temperature fluctuations. Several sensitivity runs have been performed to test the effect of small-scale temperature fluctuations on NAT nucleation. The time series of temperature fluctuations which was superimposed on the CLaMS trajectories for this purpose was based on that derived in Hoyle et al. (2005), and had mean amplitudes of $\pm 0.5 \mathrm{~K}$, similarly to those used by Engel et al. (2013), for heterogeneous ice nucleation. The results of these model runs are presented in Sect. 3.4.

\section{Model configuration used in this study}

For this study, the total number density of liquid aerosol particles was set to $15 \mathrm{~cm}^{-3}$. Half of these particles contained no foreign nuclei, and the initial radius of these particles followed a log-normal distribution, with mode radius of $70 \mathrm{~nm}$, and $\sigma=1.8$ (e.g. Dye et al., 1992). The number density of foreign nuclei was set to $7.5 \mathrm{~cm}^{-3}$, with a radius of $20 \mathrm{~nm}$. The foreign nuclei were immersed in the remaining $7.5 \mathrm{~cm}^{-3}$ liquid droplets, which were assigned a radius of $70 \mathrm{~nm}$ (equal to the mode radius of the liquid-only droplet distribution). This was done to achieve the computational efficiency necessary to run the large number of trajectories simulated. As all NAT nucleation in the simulations occurred heterogeneously, this simplification had no significant effect on the results. The composition of the liquid solution was initially in equilibrium with the gas phase, and the $\mathrm{H}_{2} \mathrm{O}$ and $\mathrm{HNO}_{3}$ mixing ratios were set at the beginning of each trajectory, using daily mean values of Microwave Limb Sounder (MLS) measurements. These daily vortex average values were calculated from the MLS data by averaging over all cloud-free points within the polar vortex on a particular day. By adjusting the model until the CALIOP PSC observations were reproduced as well as possible, the following best values were determined for the fitting parameters: $\alpha_{i}=43^{\circ}, \gamma^{\prime}=650 \mathrm{~K}^{3}$ and $P_{\text {pre }}=1 \times 10^{-6} \mathrm{deg}^{-1}$. In Sect. 3, comparisons of the observations and model calculations using the new parameterisation with these values are presented, as well as further calculations which illustrate the sensitivity of the results to changes in the fitting parameters.

\subsection{Constant NAT nucleation rate}

Experiments using constant, air-volume-based nucleation rates were also performed to provide a comparison with the new physically based NAT nucleation parameterisation. Similarly to previous studies (as summarised in Table 1), the constant nucleation rate was applied at all the trajectory points where the temperature was below $T_{\mathrm{NAT}}$. Rate coefficients of between $J_{\mathrm{NAT}}^{\text {het, vol }}=7.0 \times 10^{-6} \mathrm{~cm}^{-3} \mathrm{air} \mathrm{h}^{-1}$ and $J_{\mathrm{NAT}}^{\text {het, vol }}=2 \times 10^{-5} \mathrm{~cm}^{-3}$ air h$^{-1}$ were run along trajectories for the fourth CALIPSO overpass of the Arctic vortex on 24 December 2009 (hereafter 24_04), and the nucleation rate producing the best results, $J_{\mathrm{NAT}}^{\text {het, vol }}=9 \times 10^{-6} \mathrm{~cm}^{-3}$ air h${ }^{-1}$, was then applied to trajectories for the remaining orbits. The results of these model experiments are also shown in Sect. 3.

\section{Results}

\subsection{Constant vs. saturation-dependent nucleation rate coefficients}

The basic difference between the new parameterisation and the constant, volume-based nucleation rate can be illustrated by plotting the development of the NAT saturation ratio and the increase in NAT number density as a function of time, for a typical trajectory (taken from orbit 24_04) as in Fig. 5. The model was run once with each nucleation parameterisation, along this $60 \mathrm{~h}$ trajectory, which was initialised with a temperature of $193.05 \mathrm{~K}$ and a pressure of $25.5 \mathrm{hPa}$. The $\mathrm{HNO}_{3}$ mixing ratio of $13.5 \mathrm{ppbv}$ and water vapour mixing ratio of $5.7 \mathrm{ppmv}$ were taken from the daily average MLS data, as described in Sect. 2.3.

The constant nucleation rate was applied as soon as the temperature drops below $T_{\mathrm{NAT}}$, and therefore the number density of NAT particles began to increase at this point. The new parameterisation on the other hand led to a significant formation of NAT only after the temperature reached approximately $T_{\mathrm{NAT}}-4 \mathrm{~K}$. Shortly after this point, the temperature stabilised briefly; therefore only about $1 \times 10^{-4} \mathrm{~cm}^{-3}$ of NAT was nucleated. Subsequently, the temperature continued to decrease, and the number density of NAT nucleated with the new parameterisation climbed rapidly to approximately $1 \times 10^{-3} \mathrm{~cm}^{-3}$. The step-like nucleation behaviour seen from the new parameterisation comes about because of the differing active site qualities on the particles. Once all the particles with sufficiently good active sites have nucleated NAT at a particular NAT saturation ratio, the NAT saturation ratio must increase before the particles with the next best active sites may also cause NAT nucleation.

The saturation ratio in both simulations remained fairly similar while the air parcel was cooling; however due to the longer times available for the NAT particles to grow, after approximately $25 \mathrm{~h}$ the uptake of $\mathrm{HNO}_{3}$ by the NAT particles in the constant nucleation case led to a slightly lower 


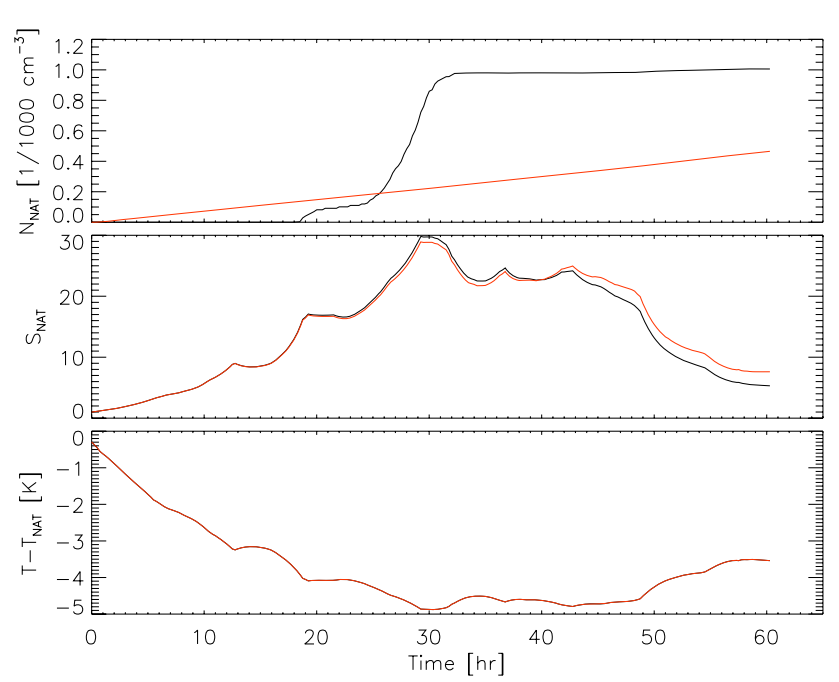

Fig. 5. A comparison of the volume-based nucleation rate parameterisation (red) and the new parameterisation (black). Both versions of the model were run along the temperature trajectory shown in the bottom panel. The development over time, of NAT number density and saturation with respect to NAT, is shown in the top and middle panels respectively.

saturation ratio than for the model with the new parameterisation. After approximately $40 \mathrm{~h}$, the NAT saturation ratio became lower for the simulation with the new parameterisation, and continued to decrease faster than in the simulation with the constant nucleation rate.

Three important differences between the constant nucleation rate and the new parameterisation are illustrated by Fig. 5. The first is that if the temperature is only slightly lower than $T_{\mathrm{NAT}}$, the constant rate will nucleate NAT particles, whereas the new parameterisation will not (0 to $20 \mathrm{~h}$ in Fig. 5). Secondly, for short periods of time at low temperatures, the new parameterisation will nucleate far more NAT than the constant rate (e.g. 20 to $30 \mathrm{~h}$ ). Finally, if the temperature remains below $T_{\mathrm{NAT}}$ for very long periods of time (as can be seen in Fig. 7, times below $T_{\text {NAT }}$ of $120 \mathrm{~h}$ or more are not unusual), the constant rate will eventually nucleate more NAT than the new parameterisation. This would happen after approximately $110 \mathrm{~h}$ for the trajectory in Fig. 5, if it were to continue without further cooling.

\subsection{Sensitivity of new parameterisation to choice of parameters}

The values of the model parameters $\gamma^{\prime}, \alpha_{0}$ and $P_{\text {pre }}$ were determined by systematically varying each parameter in turn, and comparing the resulting modelled PSC optical properties with the CALIOP observations. For this purpose, the orbit 24_04 was particularly suitable, as a fairly large, coherent area of PSC was observed, and the length of time that this air mass had experienced temperatures below $T_{\text {NAT }}$ was only between about 48 and $72 \mathrm{~h}$, minimising complications caused by mixing or by the sedimentation of PSC particles. After the match between the modelled results and the observations for orbit 24_04 was optimised, the model was applied to other orbits in a predictive capacity, to evaluate the robustness of the new parameterisation.

The PSC classifications of the model runs used to define the model parameters are shown in Fig. 6, along with contours indicating the temperature at the observation point relative to $T_{\mathrm{NAT}}$. The uppermost panel shows the observed PSC classifications. On the top row of the figure, the nucleation barrier parameter $\gamma^{\prime}$ is varied from a value of 550 to $750 \mathrm{~K}^{3}$ in steps of $50 \mathrm{~K}^{3}$. As expected at low values of $\gamma^{\prime}$, the PSC area is greater, and with increasing $\gamma^{\prime}$ the area of PSC formed gradually decreases until only those trajectories that had experienced the coldest temperatures still nucleate NAT. On the second row of Fig. 6, the nucleation strength parameter $P_{\text {pre }}$ is varied from $1 \times 10^{-7} \mathrm{deg}^{-1}$ to $1 \times 10^{-5} \mathrm{deg}^{-1}$. Here, the situation is reversed, with the larger values leading also to larger areas of PSC formed, and also an enhancement in the NAT number density, as shown by the increasing number of points being classed as Mix1/Mix2 and then as Mix-2enh. On the bottom row of the figure, the compatibility factor $\alpha_{0}$ is varied from 41 to $45^{\circ}$ in steps of $1^{\circ}$. Again, the increasing values of $\alpha_{0}$ lead to a decrease in the temperature at which NAT can form, and a reduction of the area of NAT modelled. The three plots in the middle column of Fig. 6 are identical, and represent the model results using our chosen set of parameters $\left(\alpha_{0}=43^{\circ}, P_{\text {pre }}=1 \times 10^{-6} \mathrm{deg}^{-1}\right.$ and $\left.\gamma^{\prime}=650 \mathrm{~K}^{3}\right)$.

It is evident from this plot that the same results can be achieved with different combinations of the three factors. For example, an $\alpha_{0}$ of $43^{\circ}, P_{\text {pre }}$ of $5 \times 10^{-7} \mathrm{deg}^{-1}$ and $\gamma^{\prime}$ of $650 \mathrm{~K}^{3}$ leads to a very similar PSC classification as is found when using an $\alpha_{0}$ of $45^{\circ}, P_{\text {pre }}$ of $1 \times 10^{-6} \mathrm{deg}^{-1}$ and $\gamma^{\prime}$ of $650 \mathrm{~K}^{3}$. We do not, therefore, claim that the values for the parameters which we have determined are the only values that will result in an accurate modelling of the observed PSC. However, lacking laboratory data on quantities such as the distribution of the quality of the foreign nuclei (i.e. the active site distribution), $\Delta F_{\text {diff }}$ and the surface energy of NAT, it is not possible to further constrain the choice of parameters, and the set of values which we have chosen are shown, in Figs. 7, 8 and 9, to lead to a very good representation of the observed NAT PSCs. Additionally, it can be seen in Fig. 6 that the results are relatively insensitive to small changes in the three parameters, indicating a certain robustness of the parameterisation.

In Fig. 7, PSC classifications calculated from observational data (top row) and model output from simulations using the new parameterisation (second row) as well as the constant, volume-based nucleation rate (third row) are shown. The plots represent a selection of orbits from throughout late December in which the advantage of the saturationdependent nucleation rate parameterisation over a constant rate is particularly evident. The bottom two rows of Fig. 7 show the time that the air parcel spent at temperatures below 


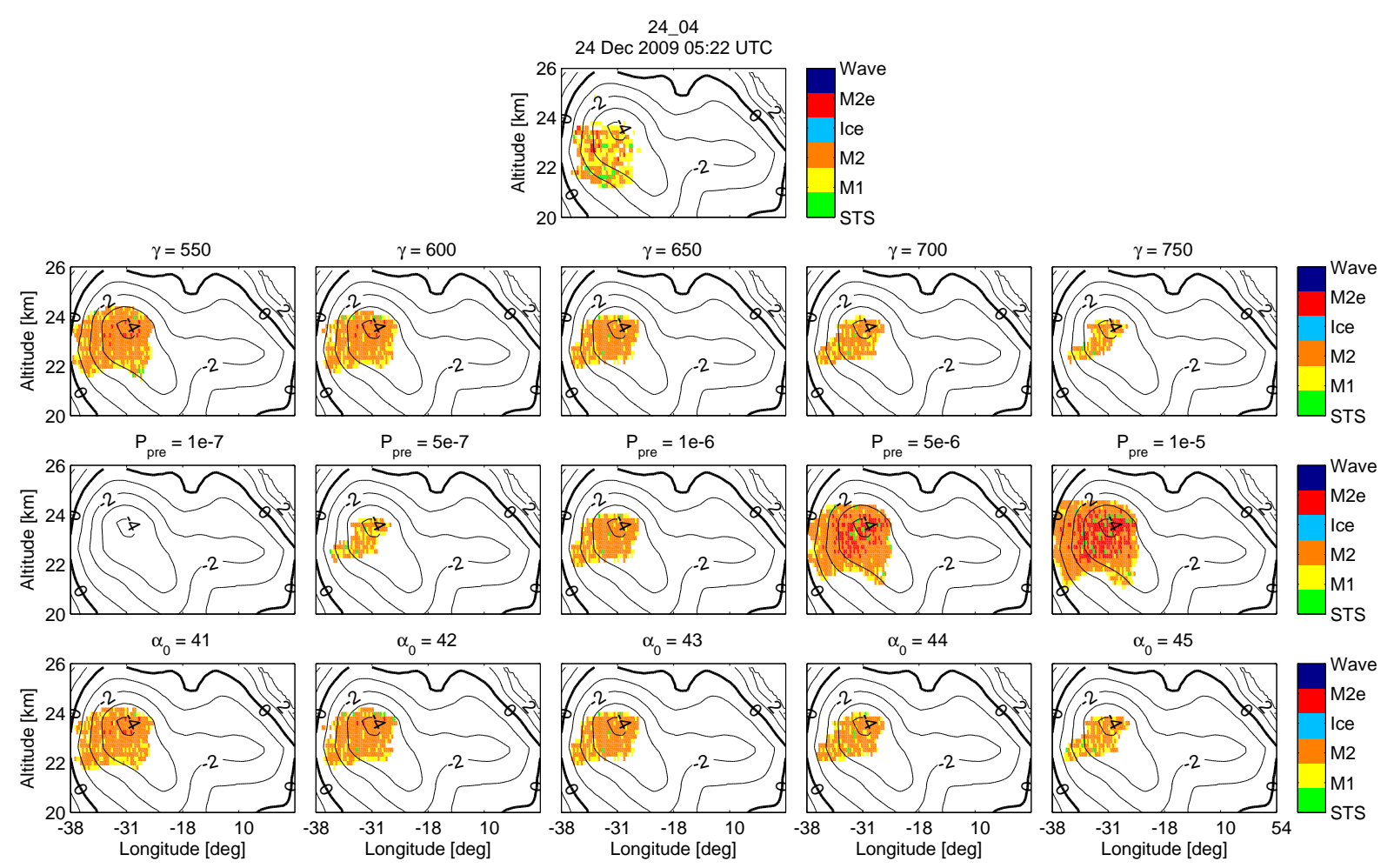

Fig. 6. An illustration of the sensitivity of the modelled NAT classification (the different classifications are indicated by the colours in the plot) to the three fitting parameters used in the new model, applied to CALIPSO orbit 24-12-2009 05:22:08 UTC (denoted orbit 24_04 in the text). The uppermost panel shows the observed PSC, and the contour lines show the temperatures with respect to $T_{\mathrm{NAT}}$ (from the ERAInterim reanalysis) at the time of CALIOP observation. The top row of panels shows how the results change as $\gamma^{\prime}$ is varied, while $P_{\text {pre }}$ is held constant at $1 \times 10^{-6} \mathrm{deg}^{-1}$, and $\alpha_{0}$ at $43^{\circ}$. In the second row, $P_{\text {pre }}$ is varied while $\gamma^{\prime}$ is held constant at $650 \mathrm{~K}^{3}$, and $\alpha_{0}$ at $43^{\circ}$, while in the bottom row $P_{\text {pre }}$ and $\gamma^{\prime}$ are held constant at $1 \times 10^{-6} \mathrm{deg}^{-1}$ and $650 \mathrm{~K}^{3}$ respectively, while $\alpha_{0}$ is varied.

$T_{\mathrm{NAT}}$ and below $T_{\mathrm{NAT}}-4 \mathrm{~K}$, while the contour lines on these two rows of plots show the minimum temperature reached along the trajectory, with respect to $T_{\mathrm{NAT}}$.

Orbit 24_04. The middle column of Fig. 7 shows the results for orbit 24_04, which, as described above, was used to fit the model parameterisations. The observed PSC consisted of a compact area of Mix 1 and Mix2, with a few spots of Mix2-enh and STS. The new parameterisation reproduces the observed area of PSC rather well; however Mix2 is overrepresented, and there are only a few areas of Mix 1 about the periphery of the cloud. The constant nucleation rate produces considerably less PSCs than observed, and at a lower altitude than the observed PSC. Of course a larger area of PSC could have been formed with a higher constant rate; however other orbits shown in Fig. 7 illustrate that a higher rate will not improve the overall agreement between the constant rate simulations and the observed PSCs.

Orbit 19_08. In the first column of Fig. 7, results for orbit 19_08 are shown. These were among the first observations of PSC for the 2009/2010 winter, and the observed area of Mix 1 and Mix2 PSC is small. The new parameterisation captures this well, also producing a small speck of PSC in the correct general area of the plot, while the constant nucleation rate, despite being on the low side for orbit 24_04, produces far too great an area of PSC. The reason for this can be seen by examining the temperature plots for this orbit. While a large number of trajectories spent up to approximately $100 \mathrm{~h}$ below $T_{\mathrm{NAT}}$, only a very small fraction reached temperatures below $T_{\mathrm{NAT}}-4 \mathrm{~K}$. As seen in Fig. 5, while the constant nucleation rate begins to produce NAT at a slow rate as soon as the temperature is below $T_{\mathrm{NAT}}$, the new parameterisation requires higher saturations and, once these saturations are reached, produces NAT relatively quickly. Of the studies discussed above, which used an air-volume-based nucleation rate, only Grooß et al. (2005) carried out any simulations where a certain NAT saturation ratio had to be exceeded before NAT was nucleated. In their case, a constant nucleation rate was applied as soon as the temperature was below $T_{\mathrm{NAT}}$, to form a low number density mode of large, sedimenting particles, while a mode of higher number density and smaller, nonsedimenting particles was nucleated only once the NAT saturation ratio exceeded 30 (equivalent to approximately $T_{\mathrm{NAT}}$ $-5 \mathrm{~K})$. Applying such a nucleation barrier may slightly improve the agreement of a simple volume-based nucleation 


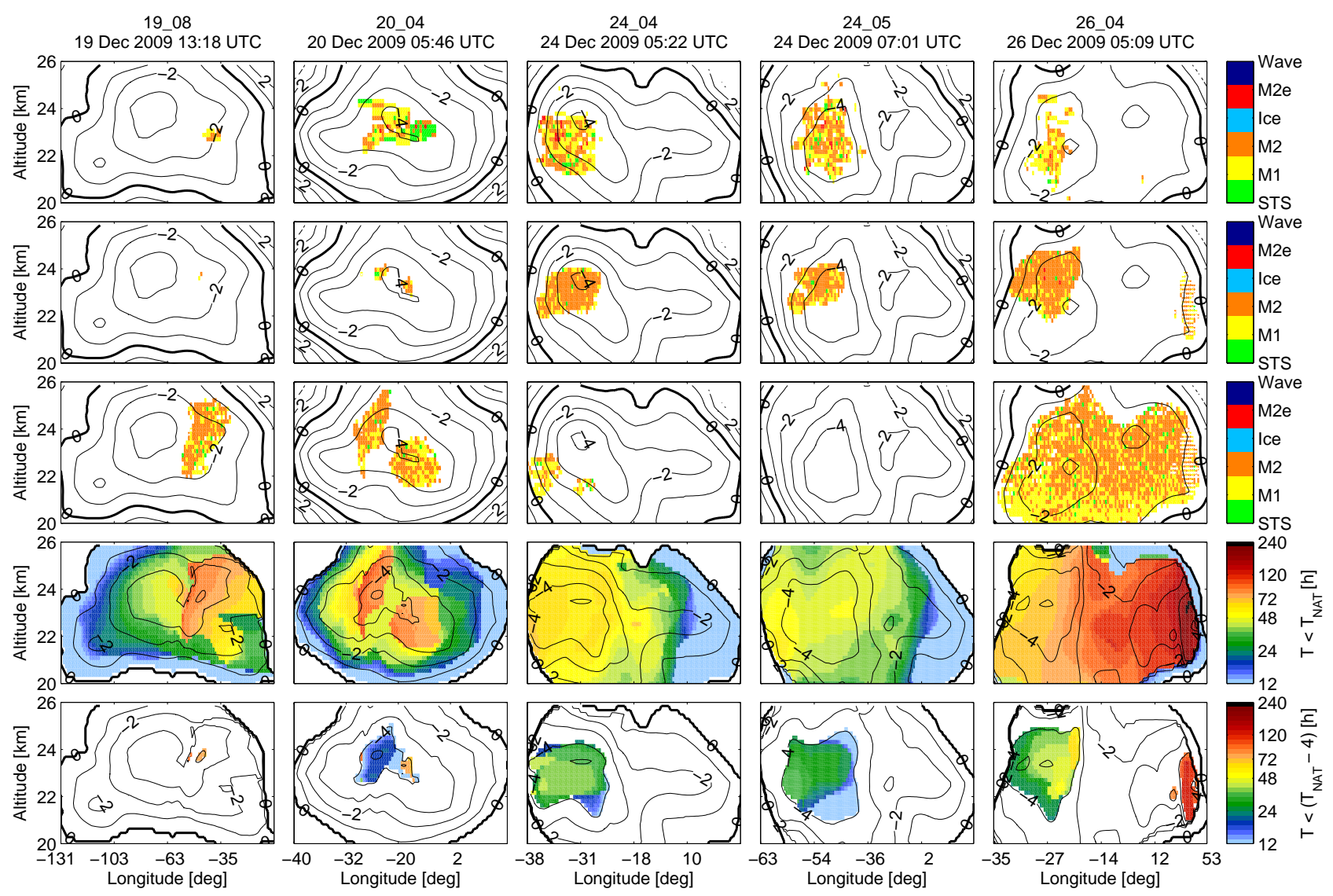

Fig. 7. A comparison of the PSC classifications calculated from CALIOP observations (top row), from model results using the new nucleation parameterisation (second row) and from model results using a constant, volume-based nucleation rate (third row). The contour lines in the upper three rows indicate the temperatures with respect to $T_{\mathrm{NAT}}$ (from the ERA-Interim reanalysis) at the time of CALIOP observation. Each column shows the results from one orbit. The bottom two rows of plots show the time spent by the trajectories at temperatures lower than $T_{\mathrm{NAT}}$ (fourth row) and $T_{\mathrm{NAT}}-4 \mathrm{~K}$ (bottom row). On these two rows, the contour lines show the minimum temperature encountered along the trajectory, with respect to $T_{\mathrm{NAT}}$.

rate with the observations in some situations; however it will not solve the fundamental problem that such a nucleation parameterisation cannot reproduce both PSCs formed during short periods at cold temperatures and PSCs formed during long periods at relatively warm temperatures.

Orbit 20_04. In the next column, results from the orbit 20_04 are shown, where the new parameterisation this time does not do as well, underestimating the area of PSC formed. The results from the constant nucleation rate are, however, again an overestimate of the PSC area. Orbit 20_04 is rather unique, in that the time spent at low temperatures is very short compared to the other orbits. The observed PSCs are mostly STS and Mix 1 clouds, an aspect which is missing in the model results, which suggests that the ERA-Interim temperatures along this orbit are very likely too warm.

Orbit 24_05. For this orbit, the new parameterisation produces PSCs at the correct altitude and longitude in comparison with the observed PSCs, albeit a slightly too small area, while the constant nucleation rate leads to a total absence of detectable PSC, due to the short time the trajectories spent at temperatures below $T_{\mathrm{NAT}}$.

Orbit 26_04. For this orbit, the constant nucleation rate leads again to a far greater area of PSC than that which was observed. The new parameterisation simulates a slightly too large area of PSC, again with an overestimation of the fraction of Mix2 with respect to Mix1. While only a few pixels of PSCs were observed at higher longitudes, the new parameterisation leads to a significant region of a Mix1/Mix2 cloud. However, these simulated trajectories have spent more than $80 \mathrm{~h}$ below $T_{\mathrm{NAT}}-4 \mathrm{~K}$ before reaching the orbit path. As discussed above, these particles will have sedimented and mixed into air parcels with different saturations ratios, which is not taken into account in the analysis. This area has therefore been marked by shading. 


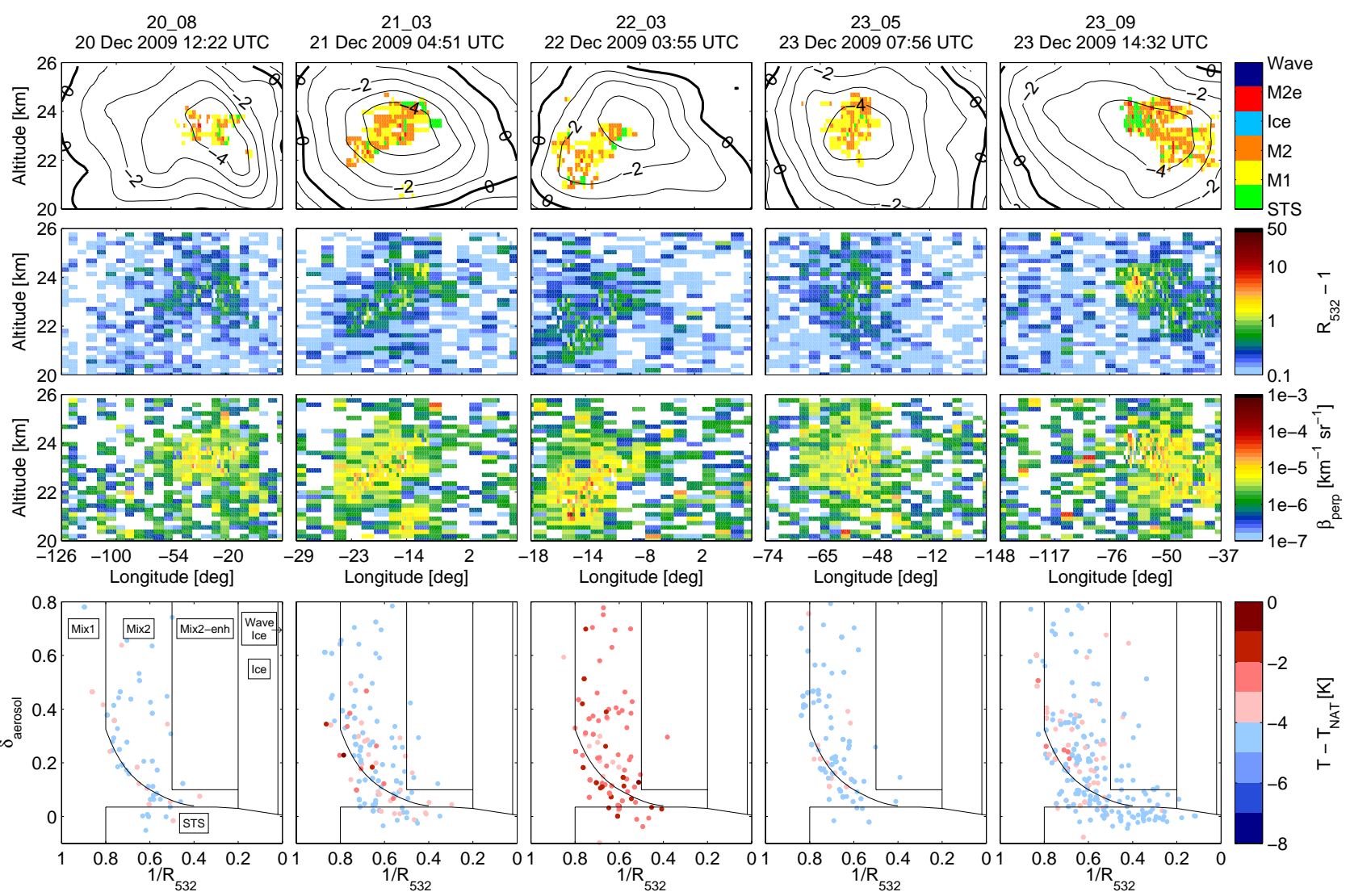

Fig. 8. PSC classification (upper row) and optical properties obtained by CALIOP along several orbits in December 2009. The contour lines in the upper row show the temperatures with respect to $T_{\mathrm{NAT}}$ (from the ERA-Interim reanalysis). The second row shows the aerosol backscatter ratio $\left(R_{532}-1\right)$ and the third the perpendicular backscatter coefficient $\left(\beta_{\text {perp }}\right)$. In the panels on the bottom row, each observation point is represented by a dot, placed according to the observed aerosol depolarisation $\left(\delta_{\text {aerosol }}\right)$, and inverse backscatter ratio, measured at a wavelength of $532 \mathrm{~nm}\left(1 / R_{532}\right)$. The points are colour coded to indicate the temperature with respect to $T_{\mathrm{NAT}}$ at the observation point.

\subsection{Modelling the NAT formation in December 2009}

The ability of the new NAT nucleation parameterisation to reproduce the observed PSC characteristics is further illustrated by comparing the results from simulating a range of orbits from mid- to late December 2009. In Fig. 8, each column shows the classification and observed optical characteristics of PSCs observed along a particular orbit. The corresponding model results are shown in Fig. 9. The model reproduces the PSC classes quite well in all orbits, both in terms of the location and the size of the modelled PSC area, and largely also in terms of optical characteristics. However, the model slightly underestimates the observed proportion of STS. This underestimation would be reduced if the temperatures were lower.

In the plots of aerosol backscatter ratio $\left(R_{532}-1\right)$, the observations exhibit a larger variability than the modelled results, with the observational data containing some points reaching values of up to about 5-6 (i.e. $1 / R_{532}>0.15$ ), while the modelled values are generally below 1 (i.e. $1 / R_{532}>0.5$ ).
The observations show perpendicular backscatter coefficients $\left(\beta_{\text {perp }}\right)$ up to $1 \times 10^{-5} \mathrm{~km}^{-1} \mathrm{sr}^{-1}$ scattered throughout the observation area, while in the modelled case such high values are confined to the vicinity of PSCs, and the modelled background signal remains below $1 \times 10^{-6} \mathrm{~km}^{-1} \mathrm{sr}^{-1}$. The cause of this difference is that the instrumental noise spikes in the background observations, particularly those which form the tails of the $\beta_{\text {perp }}$ distribution, are not entirely reproduced by the function used to generate the instrumental noise which was added to the modelled optical values. The noise in the measured backscatter is often caused by variations in radiation from space, to which the satellite-based instruments are subjected.

Comparing the bottom panels of Figs. 8 and 9 shows that while the model generally reproduces the distribution of the points as a function of $\delta_{\text {aerosol }}$ and inverse backscatter ratio, the distribution of the modelled points is more compact with respect to the inverse backscatter ratio than is the case for the observational data. While the points at small backscatter ratios $\left(1 / R_{532}>0.5\right)$ are well represented, the model misses the observed large backscatter ratios. This concerns mainly 


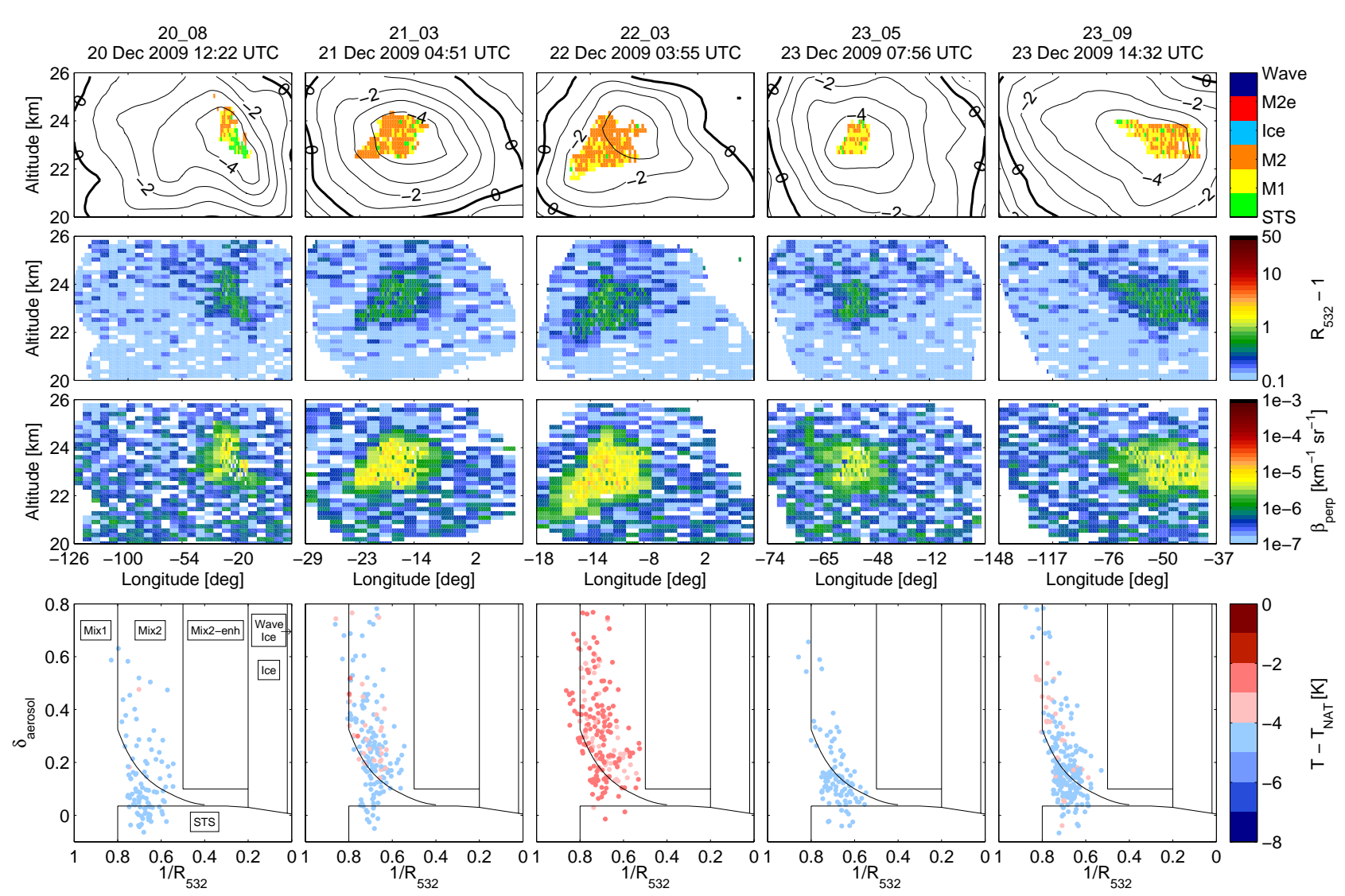

Fig. 9. As for Fig. 8, except that in this case the model results are presented.

STS clouds. Since STS clouds, owing to their large surface area, respond to temperature changes without significant kinetic delay, they act as thermometer; i.e. the only reasonable explanation is that the ERA-Interim reanalysis temperatures are occasionally too high. In the critical temperature regime of maximum $\mathrm{HNO}_{3}$ uptake by STS, a temperature reduction of just $1 \mathrm{~K}$ can cause STS clouds to grow by up to a factor of 4 in volume (Carslaw et al., 1994), i.e. about a factor 1.6 in radius, which translates into an increase in $\left(R_{532}-1\right)$ by up to a factor 6 (Biele et al., 2001). A sensitivity test, in which the temperature along the entire length of the trajectories was reduced by $1 \mathrm{~K}$, led to inverse backscatter ratios which spanned the range of the observations, and also to an increased area of the modelled PSC (not shown). Increasing the total number of aerosol droplets in the model which did not contain foreign nuclei from 7.5 to $15 \mathrm{~cm}^{-3}$, on the other hand, increases the proportion of PSC classified as STS, but does not significantly increase the modelled backscatter ratios. The uncertainty of the ERA-Interim stratospheric temperatures is of the order of $1 \mathrm{~K}$ during the 2009/2010 Arctic winter, as a detailed comparison with radiosondes launched from Ny Ålesund and Sodankylä reveals (during the RECONCILE and LAPBIAT-II (Lapland Atmosphere-Biosphere Facility) campaigns, not shown here). This corroborates the notion that the lack of high backscatters is most likely due to uncertainties in the reanalysis data.

\subsection{The effect of temperature fluctuations}

The model runs shown in Fig. 9 were repeated; however this time, random phases of the temperature fluctuations described in Sect. 2.3 were superimposed on the trajectories along which ZOMM was run. As expected, due to the increased NAT saturation ratios resulting from the temperature fluctuations, the area of the modelled NAT PSCs increased, as did the number density of the NAT particles in the clouds. In order to restore agreement with the observed properties of the PSCs, the value of $\gamma^{\prime}$ was increased from 650 to $700 \mathrm{~K}^{3}$, increasing the NAT nucleation barrier. The results of these model runs are shown in Fig. 10. Comparing Fig. 10 with Fig. 8, it can be seen that the areas and classifications of PSCs modelled when accounting for temperature fluctuations generally agree very well with the observed PSC properties, although for orbits 21_03 and 22_03 the modelled PSC areas in Fig. 10 are slightly larger than observed. However, including the fluctuations also leads to a better representation of the observed $R_{532}-1$ and $\beta_{\text {perp }}$ values, particularly in the case of orbits 20_08 and 23_05. Because of the effect of temperature fluctuations on the modelled PSC classifications, it is 


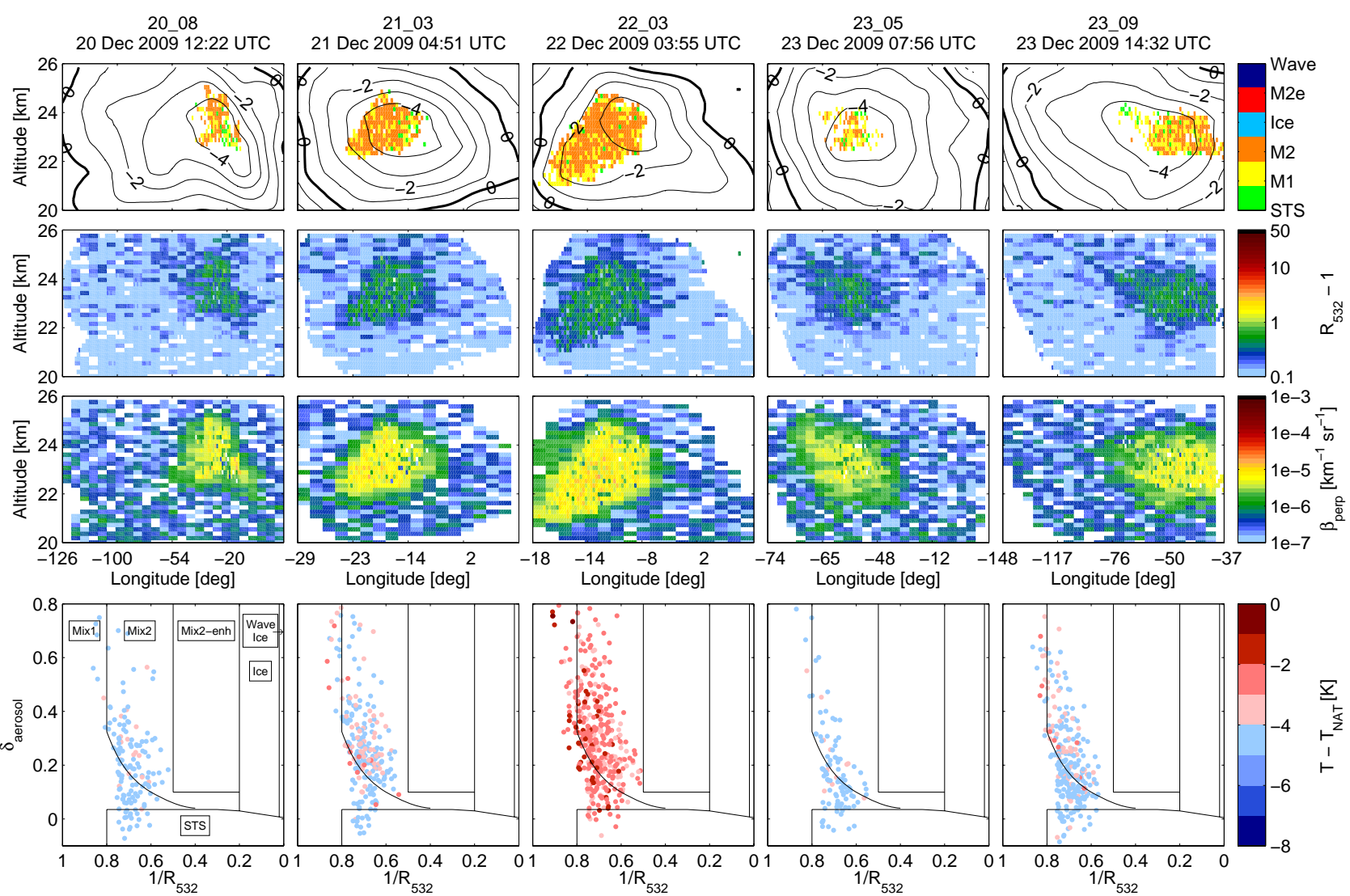

Fig. 10. As for Fig. 9, except that in this case the model results were calculated along trajectories with small-scale temperature fluctuations included, and $\gamma^{\prime}$ was set to $700 \mathrm{~K}^{3}$.

Table 2. The parameters to be used in the NAT nucleation parameterisation, for models which include a representation of the atmospheric dynamics leading to small-scale temperature fluctuations, and for models which do not include such a representation.

\begin{tabular}{lccc}
\hline Model dynamics & $\gamma^{\prime}\left(\mathrm{K}^{3}\right)$ & $P_{\text {pre }}\left(\mathrm{deg}^{-1}\right)$ & $\alpha_{0}$ \\
\hline No fluctuations & 650 & $1 \times 10^{-6}$ & $43^{\circ}$ \\
Including fluctuations & 700 & $1 \times 10^{-6}$ & $43^{\circ}$ \\
\hline
\end{tabular}

useful to define two sets of model parameters, as shown in Table 2. The first set are for use in models where small-scale air motions and the associated temperature fluctuations are not explicitly accounted for. These parameters reproduce the effect of the temperature fluctuations on NAT formation, to a large extent. The second set of parameters is suitable for models where the temperature fluctuations are explictly represented.

\section{Summary and conclusion}

The 2009/2010 Arctic winter was unique, in that during the second half of December the stratospheric temperatures were below $T_{\mathrm{NAT}}$, while having stayed at least $2 \mathrm{~K}$ above the frost point and $5 \mathrm{~K}$ above the temperature required for homogeneous ice nucleation. Whereas there have been previous studies which have suggested that nucleation on ice is not the only NAT formation pathway in the polar stratosphere, the CALIOP measurements presented by Pitts et al. (2011) and modelled here provide unprecedented evidence for persistent ice-free NAT formation on the synoptic scale. Because of the meteorological situation, the CALIOP observational data set, together with a microphysical model, could be used to perform a detailed modelling of NAT formation in the absence of ice, enabling the derivation of a parameterisation of the heterogeneous nucleation process. A new NAT nucleation parameterisation was constructed, whereby NAT nucleates on foreign nuclei, such as meteoritic dust particles, which are immersed in stratospheric aerosol droplets. The parameterisation uses active site theory to account for the range of qualities of the nucleation sites on the available nuclei. It extends the parameterisation used by Luo et al. (2003) for NAT 
formation via deposition nucleation on the uncoated surface of ice particles.

The PSC model ZOMM, containing the new parameterisation, was applied to ten orbits between 19 December 2009, when the first pixels of PSC were observed by CALIPSO, and 26 December 2009. Later orbits were excluded as they were getting close to the date when ice was first observed and also because the time that a substantial fraction of the trajectories spent below $T_{\text {NAT }}$ was longer than $80 \mathrm{~h}$, increasing the influence of factors other than nucleation on the reproducibility of the observed PSC.

It has been shown that the new NAT nucleation parameterisation leads to an accurate modelling of the observed PSC throughout this period, with the extent of the observed NAT PSC being extremely well reproduced. There is one notable exception, orbit 20_04, where the area of PSC was underestimated by the model. In this particular case, there was a relatively small number of trajectories which had spent a short time (less than $24 \mathrm{~h}$ ) at low temperatures $\left(T_{\mathrm{NAT}}-4 \mathrm{~K}\right)$. Under such conditions, small differences between the actual meteorological situation and the ERA-Interim data used by the model along the trajectories may lead to a larger apparent discrepancy between the modelled and observed PSC classifications. An examination of the modelled backscatter shows that it just marginally misses the CALIOP PSC classification criteria.

The classification of the modelled PSCs corresponds very well with the classification of the observed PSCs; however the model has a slight tendency to over-represent Mix2, and underestimate Mix1 and STS. Again this could be due to slightly too high temperatures in the trajectories. On the other hand, Engel et al. (2013) found that using the ECMWF operational analysis to calculate the trajectories, rather than the ERA-Interim data, would have led to temperatures along the trajectories having a low bias. Further, it should be noted that although the classification Mix2 is slightly over represented in the model results, the inverse backscatter values derived from both the modelled and observed PSCs straddle the boundary between Mix 1 and Mix 2 and are actually very similar (e.g. bottom rows of Figs. 8 and 9). Therefore, small shifts in inverse backscatter lead to different modelled and observed classifications of the PSC despite very similar optical properties.

The model also reproduces the individual optical quantities such as the backscatter ratio $\left(R_{532}\right)$ and the perpendicular backscatter coefficient ( $\beta_{\text {perp }}$ ) very well, while the variability as well as peak values in these quantities are not quite as high as those observed. The most significant differences are in the $\beta_{\text {perp }}$ outside of the areas where PSC has been detected. In these places the observed $\beta_{\text {perp }}$ exhibits a substantially larger variability than the modelled values. However, as no PSCs were detected in these areas, this has no impact on our results, and is most likely due to instrumental noise.

Small-scale temperature fluctuations, resulting for example from gravity waves, with horizontal wavelengths that are not resolved in the ERA-Interim data, are expected to be present in the polar stratospheres, and indeed have been the topic of many previous PSC studies (e.g. Fueglistaler et al., 2002; Luo et al., 2003; Mann et al., 2005; Höpfner et al., 2006; Eckermann et al., 2009; Alexander et al., 2011). When such fluctuations are added to the trajectories along which ZOMM is run, higher NAT saturation ratios are reached, and thus, on average, NAT nucleates at higher temperatures. However, a similar effect can also be achieved to a large extent with a lower NAT nucleation barrier $\left(\gamma^{\prime}\right)$ in the new parameterisation. The standard value of $650 \mathrm{~K}^{3}$ which was determined for $\gamma^{\prime}$ can therefore be viewed, to a certain extent, as including the main effects of the temperature fluctuations. On the other hand, if the new parameterisation were to be implemented in a model where the small-scale temperature fluctuations are resolved, a value of $\gamma^{\prime}=700 \mathrm{~K}^{3}$ should be used instead.

The new parameterisation has significant advantages over an approach which applies a single, saturation-independent, air-volume-based nucleation rate as soon as the temperature drops below $T_{\mathrm{NAT}}$. Such a method cannot accurately model both situations with temperatures remaining slightly below $T_{\text {NAT }}$ for an extended period of time as well as situations with temperatures substantially below $T_{\text {NAT }}$ prevailing over only short time periods. The new parameterisation copes well with both situations (see for example Fig. 7, orbits 24_05 and 26_04), while also partially reproducing the observed PSCs in more extreme cases (e.g. orbit 20_04).

The new parameterisation is computationally efficient and simple to implement in large-scale models. Beyond the application in our microphysical box model without sedimentation, we hope that the application of this parameterisation in three-dimensional simulations of denitrification and renitrification in the polar stratospheric vortex will provide the opportunity to follow the development of the NAT particles over extended time periods, from nucleation through their growth and subsequent sedimentation, all the way to final evaporation or tropospheric removal.

Acknowledgements. This work was supported by Swiss National Science Foundation (SNSF) grant numbers 200021_120175/1 and 200021_140663 as well as by the European Commission Seventh Framework Programme (FP7) under the grant number RECONCILE-226365-FP7-ENV-2008-1. Support for L. R. Poole is provided under NASA contract NNL11AA10D. Support for B. P. Luo by the project RECONCILE funded by the European Commission is gratefully acknowledged. Aura MLS gas species data were obtained through the Aura MLS website (http://mls.jpl.nasa.gov/index-eos-mls.php).

Edited by: D. Knopf 


\section{References}

Abbatt, J. P. D.: Interaction of $\mathrm{HNO}_{3}$ with water-ice surfaces at temperatures of the free troposphere, Geophys. Res. Lett., 24, 14791482, doi:10.1029/97GL01403, 1997.

Alexander, S. P., Klekociuk, A. R., Pitts, M. C., McDonald, A. J., and Arevalo-Torres, A.: The effect of orographic gravity waves on Antarctic polar stratospheric cloud occurrence and composition, J. Geophys. Res., 116, 3093-3109, doi:10.1029/2010JD015184, 2011.

Beyer, K. D., Seago, S. W., Chang, H. Y., and Molina, M. J.: Composition and freezing of aqueous $\mathrm{H}_{2} \mathrm{SO}_{4} / \mathrm{HNO}_{3}$ solutions under polar stratosopheric conditions, Geophys. Res. Lett., 21, 871874, doi:10.1029/94GL00915, 1994.

Biele, J., Tsias, A., Luo, B. P., Carslaw, K. S., Neuber, R., Beyerle, G., and Peter, T.: Nonequilibrium coexistence of solid and liquid particles in Arctic stratospheric clouds, J. Geophys. Res., 106, 22 991-23 007, doi:10.1029/2001JD900188, 2001.

Biermann, U. M., Presper, T., Koop, T., Mossinger, J., Crutzen, P. J., and Peter, T.: The unsuitability of meteoritic and other nuclei for polar stratospheric cloud freezing, Geophys. Res. Lett., 23, 1693-1696, doi:10.1029/96GL01577, 1996.

Brabec, M., Wienhold, F. G., Luo, B. P., Voemel, H., Immler, F., Steiner, P., Hausammann, E., Weers, U., and Peter, T.: Particle backscatter and relative humidity measured across cirrus clouds and comparison with microphysical cirrus modelling, Atmos. Chem. Phys., 12, 9135-9148, doi:10.5194/acp-12-9135-2012, 2012.

Carslaw, K. S., Luo, B. P., Clegg, S. L., Peter, T., Brimblecombe, P., and Crutzen, P. J.: Stratospheric aerosol growth and $\mathrm{HNO}_{3}$ gas-phase depletion from coupled $\mathrm{HNO}_{3}$ and wateruptake by liquid particles, Geophys. Res. Lett., 21, 2479-2482, doi:10.1029/94GL02799, 1994.

Carslaw, K. S., Wirth, M., Tsias, A., Luo, B. P., Dornbrack, A., Leutbecher, M., Volkert, H., Renger, W., Bacmeister, J. T., and Peter, T.: Particle microphysics and chemistry in remotely observed mountain polar stratospheric clouds, J. Geophys. Res., 103, 5785-5796, doi:10.1029/97JD03626, 1998.

Carslaw, K. S., Kettleborough, J. A., Northway, M. J., Davies, S., Gao, R. S., Fahey, D. W., Baumgardner, D. G., Chipperfield, M. P., and Kleinbohl, A.: A vortex-scale simulation of the growth and sedimentation of large nitric acid hydrate particles, J. Geophys. Res., 107, 8300, doi:10.1029/2001JD000467, 2002.

Crutzen, P. J. and Arnold, F.: Nitric-acid cloud formation in the cold Antarctic stratosphere - a major cause for the springtime ozone hole, Nature, 324, 651-655, doi:10.1038/324651a0, 1986.

Curtius, J., Weigel, R., Vössing, H.-J., Wernli, H., Werner, A., Volk, C.-M., Konopka, P., Krebsbach, M., Schiller, C., Roiger, A., Schlager, H., Dreiling, V., and Borrmann, S.: Observations of meteoric material and implications for aerosol nucleation in the winter Arctic lower stratosphere derived from in situ particle measurements, Atmos. Chem. Phys., 5, 3053-3069, doi:10.5194/acp-5-3053-2005, 2005.

Davies, S., Mann, G. W., Carslaw, K. S., Chipperfield, M. P., Kettleborough, J. A., Santee, M. L., Oelhaf, H., Wetzel, G., Sasano, Y., and Sugita, T.: 3-D microphysical model studies of Arctic denitrification: comparison with observations, Atmos. Chem. Phys., 5, 3093-3109, doi:10.5194/acp-5-3093-2005, 2005.

Dee, D. P., Uppala, S. M., Simmons, A. J., Berrisford, P., Poli, P., Kobayashi, S., Andrae, U., Balmaseda, M. A., Balsamo, G.,
Bauer, P., Bechtold, P., Beljaars, A. C. M., van de Berg, L., Bidlot, J., Bormann, N., Delsol, C., Dragani, R., Fuentes, M., Geer, A. J., Haimberger, L., Healy, S. B., Hersbach, H., Holm, E. V., Isaksen, L., Kallberg, P., Koehler, M., Matricardi, M., McNally, A. P., Monge-Sanz, B. M., Morcrette, J. J., Park, B. K., Peubey, C., de Rosnay, P., Tavolato, C., Thepaut, J. N., and Vitart, F.: The ERA-Interim reanalysis: configuration and performance of the data assimilation system, Q. J. Roy. Meteor. Soc., 137, 553-597, doi:10.1002/qj.828, 2011.

Dörnbrack, A., Pitts, M. C., Poole, L. R., Orsolini, Y. J., Nishii, K., and Nakamura, H.: The 2009-2010 Arctic stratospheric winter general evolution, mountain waves and predictability of an operational weather forecast model, Atmos. Chem. Phys., 12, 36593675, doi:10.5194/acp-12-3659-2012, 2012.

Drdla, K., Schoeberl, M. R., and Browell, E. V.: Microphysical modeling of the 1999-2000 Arctic winter: 1. Polar stratospheric clouds, denitrification, and dehydration, J. Geophys. Res., 108, 8312, doi:10.1029/2001JD000782, 2003.

Dye, J. E., Baumgardner, D., Gandrud, B. W., Kawa, S. R., Kelly, K. K., Loewenstein, M., Ferry, G., Chan, K. R., and Gary, B. L.: Particle-size distributions in Arctic polar stratospheric clouds, growth and freezing of sulfuric-acid droplets, and implications for cloud formation, J. Geophys. Res., 97, 8015-8034, 1992.

Eckermann, S. D., Hoffmann, L., Hoepfner, M., Wu, D. L., and Alexander, M. J.: Antarctic NAT PSC belt of June 2003: Observational validation of the mountain wave seeding hypothesis, Geophys. Res. Lett., 36, L02807, doi:10.1029/2008GL036629, 2009.

Engel, I., Luo, B. P., Pitts, M. C., Poole, L. R., Hoyle, C. R., Grooß, J.-U., Dörnbrack, A., and Peter, T.: Heterogeneous formation of polar stratospheric clouds - Part 2: Nucleation of ice on synoptic scales, Atmos. Chem. Phys. Discuss., 13, 8831-8872, doi:10.5194/acpd-13-8831-2013, 2013.

Farman, J. C., Gardiner, B. G., and Shanklin, J. D.: Large losses of total ozone in Antarctica reveal seasonal $\mathrm{ClO} \times \mathrm{x} / \mathrm{NO}_{\mathrm{x}}$ interaction, Nature, 315, 207-210, doi:10.1038/315207a0, 1985.

Fueglistaler, S., Luo, B.P., Voigt, C., Carslaw, K.S., and Peter, Th.: NAT-rock formation by mother clouds: a microphysical model study, Atmos. Chem. Phys., 2, 93-98, doi:10.5194/acp-2-932002, 2002.

Gary, B. L.: Mesoscale temperature fluctuations in the stratosphere, Atmos. Chem. Phys., 6, 4577-4589, doi:10.5194/acp-6-45772006, 2006.

Gary, B. L.: Mesoscale temperature fluctuations in the Southern Hemisphere stratosphere, Atmos. Chem. Phys., 8, 4677-4681, doi:10.5194/acp-8-4677-2008, 2008.

Grooß, J.-U., Günther, G., Müller, R., Konopka, P., Bausch, S., Schlager, H., Voigt, C., Volk, C.M., and Toon, G. C.: Simulation of denitrification and ozone loss for the Arctic winter 2002/2003, Atmos. Chem. Phys., 5, 1437-1448, doi:10.5194/acp-5-14372005, 2005.

Hanson, D. and Mauersberger, K.: Laboratory studies of the nitric-acid trihydrate - implications for the south polar stratosphere, Geophys. Res. Lett., 15, 855-858, doi:10.1029/GL015i008p00855, 1988.

Höpfner, M., Larsen, N., Spang, R., Luo, B. P., Ma, J., Svendsen, S. H., Eckermann, S. D., Knudsen, B., Massoli, P., Cairo, F., Stiller, G., v. Clarmann, T., and Fischer, H.: MIPAS detects Antarctic stratospheric belt of NAT PSCs caused by mountain 
waves, Atmos. Chem. Phys., 6, 1221-1230, doi:10.5194/acp-61221-2006, 2006.

Hoyle, C. R., Luo, B. P., and Peter, T.: The origin of high ice crystal number densities in cirrus clouds, J. Atmos. Sci., 62, 2568-2579, doi:10.1175/JAS3487.1, 2005.

Hoyle, C. R., Pinti, V., Welti, A., Zobrist, B., Marcolli, C., Luo, B., Hoeskuldsson, A., Mattsson, H. B., Stetzer, O., Thorsteinsson, T., Larsen, G., and Peter, T.: Ice nucleation properties of volcanic ash from Eyjafjallajokull, Atmos. Chem. Phys., 11, 9911-9926, doi:10.5194/acp-11-9911-2011, 2011.

Iraci, L. T., Middlebrook, A. M., Wilson, M. A., and Tolbert, M. A.: Growth of nitric-acid hydrates on thin sulfuric-acid films, Geophys. Res. Lett., 21, 867-870, doi:10.1029/94GL00916, 1994.

Kärcher, B. and Lohmann, U.: A Parameterization of cirrus cloud formation: Homogeneous freezing including effects of aerosol size, J. Geophys. Res., 107, 4698, doi:10.1029/2001JD001429, 2002.

Knopf, D. A., Koop, T., Luo, B. P., Weers, U. G., and Peter, T.: Homogeneous nucleation of NAD and NAT in liquid stratospheric aerosols: insufficient to explain denitrification, Atmos. Chem. Phys., 2, 207-214, doi:10.5194/acp-2-207-2002, 2002.

Koop, T., Biermann, U. M., Raber, W., Luo, B. P., Crutzen, P. J., and Peter, T.: Do stratospheric aerosol droplets freeze above the ice frost point, Geophys. Res. Lett., 22, 917-920, doi:10.1029/95GL00814, 1995.

Koop, T., Luo, B. P., Tsias, A., and Peter, T.: Water activity as the determinant for homogeneous ice nucleation in aqueous solutions, Nature, 406, 611-614, 2000.

Larsen, N., Knudsen, B. M., Svendsen, S. H., Deshler, T., Rosen, J. M., Kivi, R., Weisser, C., Schreiner, J., Mauerberger, K., Cairo, F., Ovarlez, J., Oelhaf, H., and Spang, R.: Formation of solid particles in synoptic-scale Arctic PSCs in early winter 2002/2003, Atmos. Chem. Phys., 4, 2001-2013, doi:10.5194/acp-4-20012004, 2004.

Luo, B. P., Voigt, C., Fueglistaler, S., and Peter, T.: Extreme NAT supersaturations in mountain wave ice PSCs: A clue to NAT formation, J. Geophys. Res., 108, 4441, doi:10.1029/2002JD003104, 2003.

Mann, G. W., Carslaw, K. S., Chipperfield, M. P., Davies, S., and Eckermann, S. D.: Large nitric acid trihydrate particles and denitrification caused by mountain waves in the Arctic stratosphere, J. Geophys. Res., 110, D08202, doi:10.1029/2004JD005271, 2005.

Marcolli, C., Gedamke, S., Peter, T., and Zobrist, B.: Efficiency of immersion mode ice nucleation on surrogates of mineral dust, Atmos. Chem. Phys., 7, 5081-5091, doi:10.5194/acp-7-50812007, 2007.

McKenna, D. S., Konopka, P., Grooß, J.-U., Gunther, G., Müller, R., Spang, R., Offermann, D., and Orsolini, Y.: A new Chemical Lagrangian Model of the Stratosphere (CLaMS) - 1. Formulation of advection and mixing, J. Geophys. Res., 107, 4309, doi:10.1029/2000JD000114, 2002.

Meilinger, S. K., Koop, T., Luo, B. P., Huthwelker, T., Carslaw, K. S., Krieger, U., Crutzen, P. J., and Peter, T.: Size-dependent stratospheric droplet composition in lee wave temperature fluctuations and their potential role in PSC freezing, Geophys. Res. Lett., 22, 3031-3034, doi:10.1029/95GL03056, 1995.

Middlebrook, A. M., Berland, B. S., George, S. M., Tolbert, M. A., and Toon, O. B.: Real refractive-indexes of infrared- characterized nitric-acid ice films - implications for optical measurements of polar stratospheric clouds, J. Geophys. Res., 99, 25655-25666, doi:10.1029/94JD02391, 1994.

Mishchenko, M. I.: Light-scattering by randomly oriented axially symmetrical particles, J. Opt. Soc. Am. A, 8, 871-882, doi:10.1364/JOSAA.8.000871, 1991.

Molina, M. J., Zhang, R., Wooldridge, P. J., Mcmahon, J. R., Kim, J. E., Chang, H. Y., and Beyer, K. D.: Physical chemistry of the $\mathrm{H}_{2} \mathrm{SO}_{4} / \mathrm{HNO}_{3} / \mathrm{H}_{2} \mathrm{O}$ system - implications for polar stratospheric clouds, Science, 261, 1418-1423, doi:10.1126/science.261.5127.1418, 1993.

Murphy, D. M., Cziczo, D. J., Hudson, P. K., and Thomson, D. S.: Carbonaceous material in aerosol particles in the lower stratosphere and tropopause region, J. Geophys. Res., 112, D04203, doi:10.1029/2006JD007297, 2007.

Pagan, K. L., Tabazadeh, A., Drdla, K., Hervig, M. E., Eckermann, S. D., Browell, E. V., Legg, M. J., and Foschi, P. G.: Observational evidence against mountain-wave generation of ice nuclei as a prerequisite for the formation of three solid nitric acid polar stratospheric clouds observed in the Arctic in early December 1999, J. Geophys. Res., 109, D04312, doi:10.1029/2003JD003846, 2004.

Peter, T. and Grooß, J.-U.: Chapter 4: Polar Stratospheric Clouds and Sulfate Aerosol Particles: Microphysics, Denitrification and Heterogeneous Chemistry, in: Stratospheric Ozone Depletion and Climate Change, The Royal Society of Chemistry, 108-144, doi:10.1039/9781849733182-00108, 2012.

Pinti, V., Marcolli, C., Zobrist, B., Hoyle, C. R., and Peter, T.: Ice nucleation efficiency of clay minerals in the immersion mode, Atmos. Chem. Phys., 12, 5859-5878, doi:10.5194/acp-12-58592012, 2012.

Pitts, M. C., Poole, L. R., and Thomason, L. W.: CALIPSO polar stratospheric cloud observations: second-generation detection algorithm and composition discrimination, Atmos. Chem. Phys., 9, 7577-7589, doi:10.5194/acp-9-7577-2009, 2009.

Pitts, M. C., Poole, L. R., Doernbrack, A., and Thomason, L. W.: The 2009-2010 Arctic polar stratospheric cloud season: a CALIPSO perspective, Atmos. Chem. Phys., 11, 2161-2177, doi:10.5194/acp-11-2161-2011, 2011.

Ravishankara, A. R. and Hanson, D. R.: Differences in the reactivity of Type I polar stratospheric clouds depending on their phase, J. Geophys. Res., 101, 3885-3890, doi:10.1029/95JD03009, 1996.

Salawitch, R. J., Wofsy, S. C., and Mcelroy, M. B.: Influence of polar stratospheric clouds on the depletion of Antarctic ozone, Geophys. Res. Lett., 15, 871-874, doi:10.1029/GL015i008p00871, 1988.

Seinfeld, J. H. and Pandis, S. N.: Atmospheric Chemistry and Physics, From Air Pollution to Climate Change, Wiley, 2006.

Solomon, S., Garcia, R. R., Rowland, F. S., and Wuebbles, D. J.: On the depletion of Antarctic ozone, Nature, 321, 755-758, doi:10.1038/321755a0, 1986.

Tabazadeh, A., Djikaev, Y. S., Hamill, P., and Reiss, H.: Laboratory evidence for surface nucleation of solid polar stratospheric cloud particles, J. Phys. Chem. A, 106, 10238-10246, doi:10.1021/jp021045k, 2002.

Toon, O. B., Hamill, P., Turco, R. P., and Pinto, J.: Condensation of $\mathrm{HNO}_{3}$ and $\mathrm{HCl}$ in the winter polar stratospheres, Geophys. Res. Lett., 13, 1284-1287, doi:10.1029/GL013i012p01284, 1986. 
Voigt, C., Schreiner, J., Kohlmann, A., Zink, P., Mauersberger, K., Larsen, N., Deshler, T., Kroger, C., Rosen, J., Adriani, A., Cairo, F., Di Donfrancesco, G., Viterbini, M., Ovarlez, J., Ovarlez, H., David, C., and Dornbrack, A.: Nitric acid trihydrate (NAT) in polar stratospheric clouds, Science, 290, 1756-1758, doi:10.1126/science.290.5497.1756, 2000.

Voigt, C., Schlager, H., Luo, B. P., Dörnbrack, A., Roiger, A., Stock, P., Curtius, J., Vössing, H., Borrmann, S., Davies, S., Konopka, P., Schiller, C., Shur, G., and Peter, T.: Nitric Acid Trihydrate (NAT) formation at low NAT supersaturation in Polar Stratospheric Clouds (PSCs), Atmos. Chem. Phys., 5, 13711380, doi:10.5194/acp-5-1371-2005, 2005.

von Hobe, M., Bekki, S., Borrmann, S., Cairo, F., D'Amato, F., Di Donfrancesco, G., Dörnbrack, A., Ebersoldt, A., Ebert, M., Emde, C., Engel, I., Ern, M., Frey, W., Genco, S., Griessbach, S., Grooß, J.-U., Gulde, T., Günther, G., Hösen, E., Hoffmann, L., Homonnai, V., Hoyle, C. R., Isaksen, I. S. A., Jackson, D. R., Jánosi, I. M., Jones, R. L., Kandler, K., Kalicinsky, C., Keil, A., Khaykin, S. M., Khosrawi, F., Kivi, R., Kuttippurath, J., Laube, J. C., Lefèvre, F., Lehmann, R., Ludmann, S., Luo, B. P., Marchand, M., Meyer, J., Mitev, V., Molleker, S., Müller, R., Oelhaf, H., Olschewski, F., Orsolini, Y., Peter, T., Pfeilsticker, K., Piesch, C., Pitts, M. C., Poole, L. R., Pope, F. D., Ravegnani, F., Rex, M., Riese, M., Röckmann, T., Rognerud, B., Roiger, A., Rolf, C., Santee, M. L., Scheibe, M., Schiller, C., Schlager, H., Siciliani de Cumis, M., Sitnikov, N., Søvde, O. A., Spang, R., Spelten, N., Stordal, F., Sumińska-Ebersoldt, O., Ulanovski, A., Ungermann, J., Viciani, S., Volk, C. M., vom Scheidt, M., von der Gathen, P., Walker, K., Wegner, T., Weigel, R., Weinbruch, S., Wetzel, G., Wienhold, F. G., Wohltmann, I., Woiwode, W., Young, I. A. K., Yushkov, V., Zobrist, B., and Stroh, F.: Reconciliation of essential process parameters for an enhanced predictability of Arctic stratospheric ozone loss and its climate interactions (RECONCILE): activities and results, Atmos. Chem. Phys., 13, 92339268, doi:10.5194/acp-13-9233-2013, 2013.
Wirth, M., Tsias, A., Dornbrack, A., Weiss, V., Carslaw, K. S., Leutbecher, M., Renger, W., Volkert, H., and Peter, T.: Modelguided Lagrangian observation and simulation of mountain polar stratospheric clouds, J. Geophys. Res., 104, 23971-23981, doi:10.1029/1998JD100095, 1999.

Zobrist, B., Soonsin, V., Luo, B. P., Kreiger, K., Marcolli, C., Peter, T., and Koop, T.: Ultra-slow water diffusion in aqueous sucrose glasses, Phys. Chem. Chem. Phys., 13, 3514-3526, doi:10.1039/c0cp01273d, 2011. 